FOMENTO DE LOS HÁBITOS DE VIDA SALUDABLE EN EL CONTADOR PÚBLICO A PARTIR DESDE LA PRÁCTICA PROFESIONAL

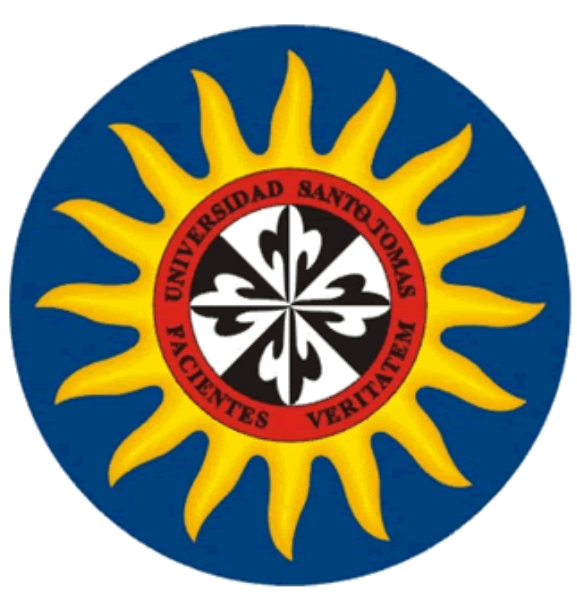

DANIEL ANDRES GARZON CESPEDES

UNIVERSIDAD SANTO TOMAS

FACULTAD DE CONTADURIA PÚBLICA

VILLAVICENCIO

2019 
FOMENTO DE HÁBITOS DE VIDA SALUDABLE EN EL CONTADOR PÚBLICO A PARTIR DESDE SU PRÁCTICA PROFESIONAL

DANIEL ANDRES GARZON CESPEDES

Trabajo de grado presentado como requisito para optar por el título de Contador Público

\author{
Director \\ LINDEY ANGELICA GOMEZ SUAREZ \\ Contador Público
}

UNIVERSIDAD SANTO TOMAS

FACULTAD DE CONTADURIA PÚBLICA

VILLAVICENCIO 


\section{Autoridades Académicas}

P. José Gabriel MESA ANGULO, O. P. Rector General

\section{P. Eduardo GONZÁLEZ GIL, O. P.}

Vicerrector Académico General

\section{P. José Antonio BALAGUERA CERPADA O.P.}

Rector Sede Villavicencio

\section{P. Rodrigo GARCÍA JARA, O.P.}

Vicerrector Académico Sede Villavicencio

\section{Mg.JULIETH ANDREA SIERRA TOBÓN}

Secretaria de División Sede Villavicencio

\section{JAVIER MAURICIO ALZATE TABARES}

Decano Facultad de Contaduría Pública. 
Nota De Aceptación

JAVIER MAURICIO ALZATE TABARES

Decano de Facultad de Contaduría Pública

LINDEY ANGELICA GOMEZ SUAREZ

Director Trabajo de Grado

MILTON ALEJANDRO TRIANA

Jurado

TATIANA ALEJANDRA GARZÓN

Jurado

Villavicencio, octubre de 2019 


\section{Contenido}

Pág.

Resumen

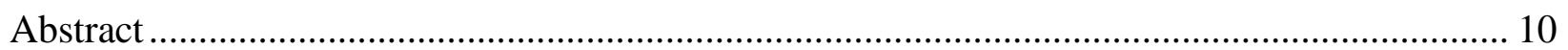

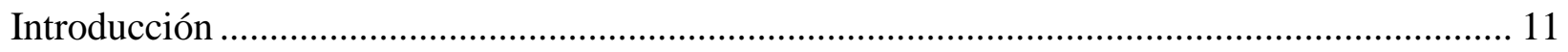

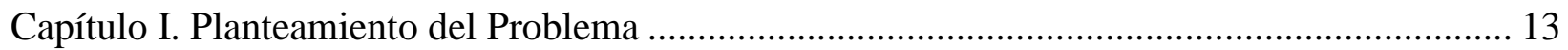

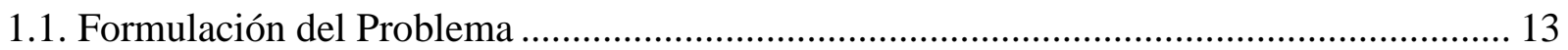

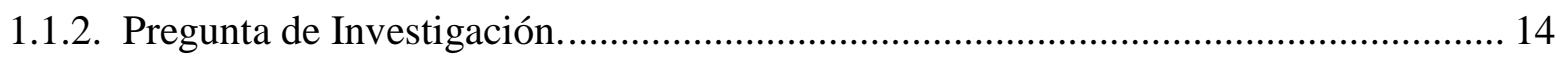

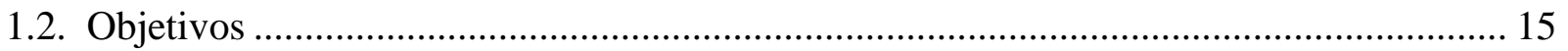

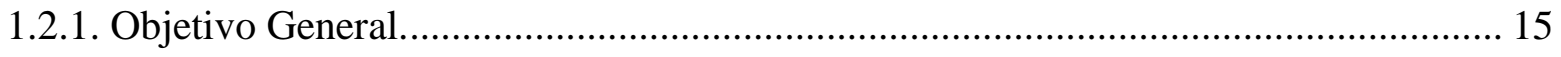

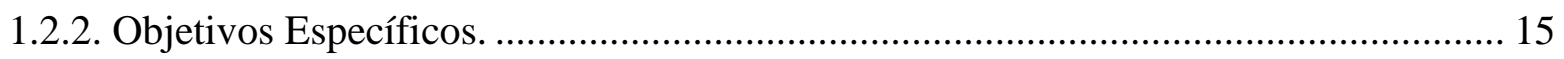

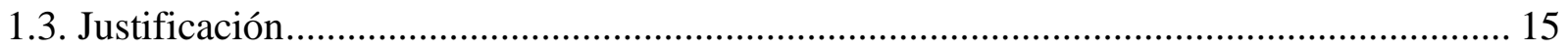

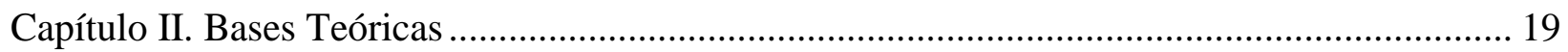

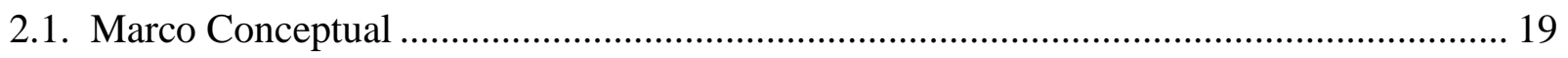

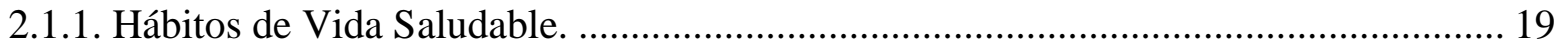

2.1.2. Enfermedades Crónicas o No Transmisibles (ENT). ................................................. 20

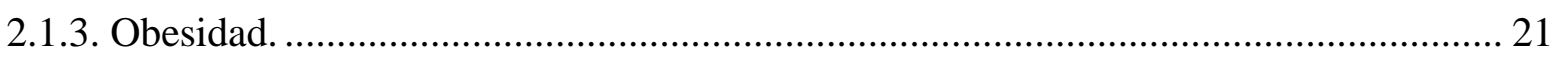

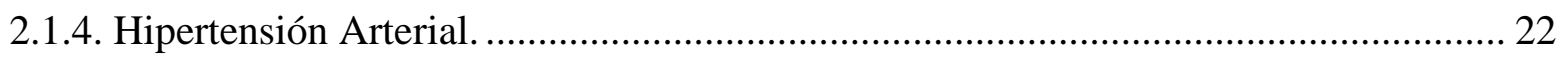

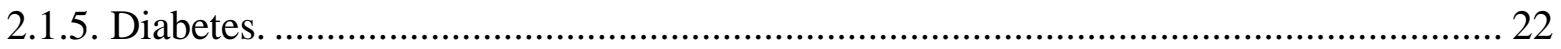

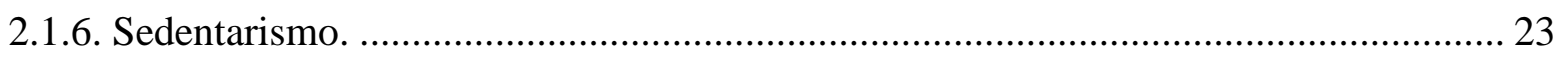

2.1.7. Ataque Cardiaco (Infarto Miocárdico).................................................................... 24

2.1.8. Nutrición: Alimentación Saludable y Balanceada.......................................................... 24

2.1.9. La Importancia del Sueño.................................................................................. 25

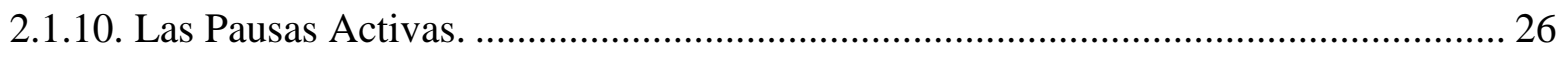

2.1.11. Estrategias Pedagógicas............................................................................................ 26

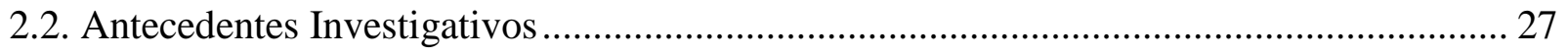

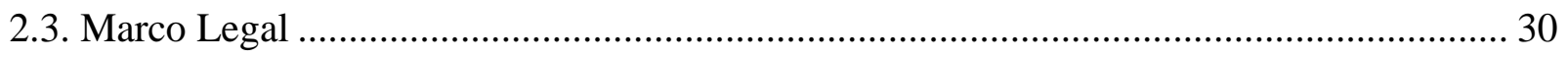

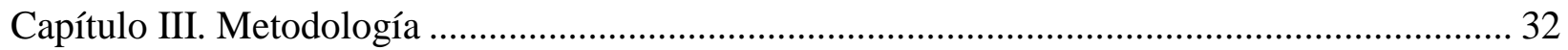

3.1. Enfoque, Alcance y Diseño …………………………................................................... 32 


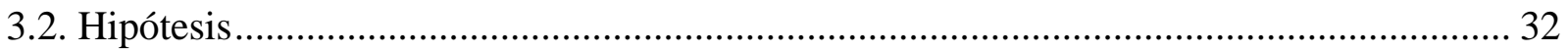

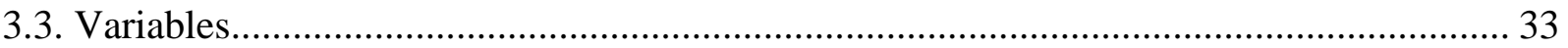

3.4. Población y Muestra............................................................................................. 34

3.5. Técnicas e Instrumento de Recolección de Datos ....................................................... 34

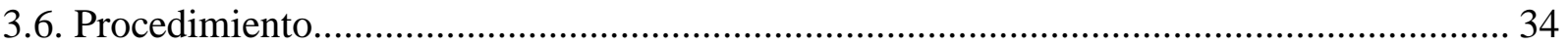

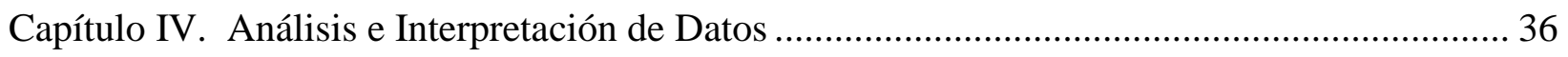

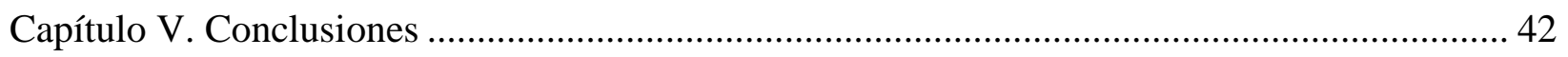

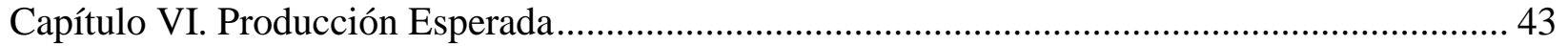

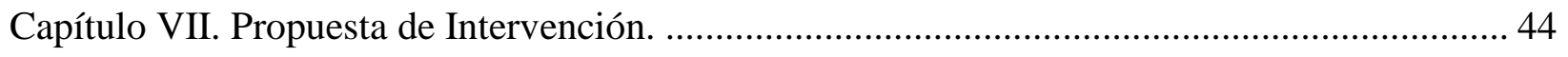

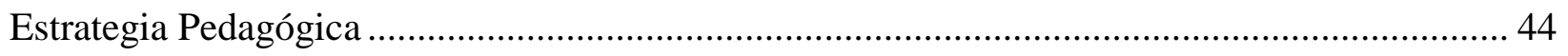

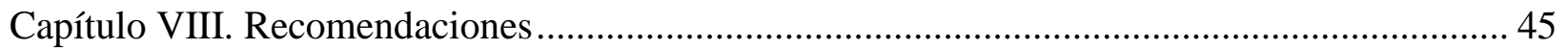

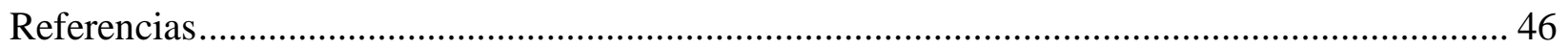

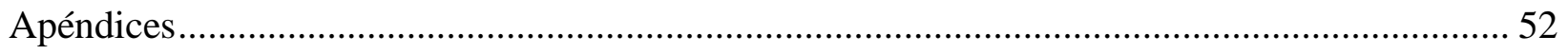




\section{Índice de Gráficos}

Pág.

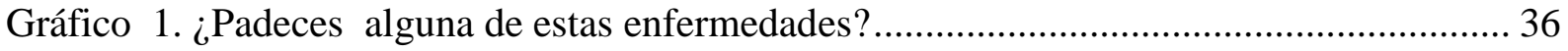

Gráfico 2. ¿Tienes antecedentes familiares de infarto?........................................................... 37

Gráfico 3. ¿Cuánta fruta consumes al día?............................................................................ 37

Gráfico 4. ¿En tu jornada laboral realizas pausas activas? ...................................................... 38

Gráfico 5. ¿Cuántas veces consumes verduras a la semana? .................................................... 39

Gráfico 6. ¿Qué cantidad de agua consumes al día? ………………………………........... 39

Gráfico 7. ¿Compartes con tus seres queridos al menos dos horas diarias? ............................ 40

Gráfico 8. ¿Cuántas horas consideras que duermes al día?....................................................... 41

Gráfico 9. ¿Lava sus manos antes de consumir algún alimento?............................................... 41 


\section{Índice de Apéndices}

Pág.

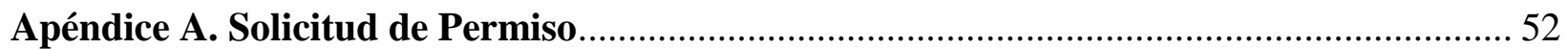

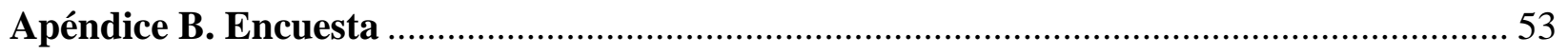

Apéndice C. Guía "Hábitos y Estilos de Vida Saludables. guía para Contadores Públicos 54 


\section{Resumen}

El presente estudio es el resultado de una investigación que tiene como objeto diseñar una estrategia pedagógica (guía) que incluya pautas y lineamientos prácticos para propiciar la realización de actividades diarias tendientes a obtener hábitos saludables en los egresados de Contaduría Pública de la Universidad Santo Tomás sede Villavicencio, permitiéndoles así, mejorar su estilo de vida y ambiente laboral.

La metodología se plantea desde el enfoque mixto (cualitativo y cuantitativo), alcance descriptivo, y parte de literatura relacionada con los factores que contribuyen al desarrollo de estilos de vida saludable, junto con aquellos asociados al deterioro de los mismo, trayendo como consigo el detrimento de la salud y el aumento de la morbimortalidad. Luego de la aplicación de instrumentos, se hace un análisis de la información recolectada. Dentro de los resultados se obtiene que la población a estudio carece de hábitos de vida saludables, asociados a parámetros como alimentación saldable, jornadas laborales, pausas activas, tiempo en familia y para dormir. De tal modo se recomienda aplicar un manual que contenga información y actividades especificas para este grupo poblacional y que propenda por el bienestar integral y el desarrollo biopsicosocial de los Contadores Públicos, llevando estilos de vida saludables.

Palabras Clave. Contador Público; Enfermedades No Transmisibles; Factores de Riesgo; Hábitos Saludables; Entorno Laboral. 


\begin{abstract}
The present study is the result of a research that aims to design a pedagogical strategy (guide) that includes guidelines and practical guidelines to encourage the performance of daily activities aimed at obtaining healthy habits in the public accounting graduates of the Santo Tomás University. Villavicencio, allowing them to improve their lifestyle and work environment.

The methodology is proposed from the mixed approach (qualitative and quantitative), descriptive scope, and part of literature related to the factors that contribute to the development of healthy lifestyles, along with those associated with the deterioration of them, bringing with it the detriment of health and the increase in morbidity and mortality. After the application of instruments, an analysis of the information collected is made. The results show that the study population lacks healthy lifestyle habits, associated with parameters such as healthy eating, working hours, active breaks, family time and sleep. In this way, it is recommended to apply a manual that contains information and specific activities for this population group that promotes the integral wellbeing and biopsychosocial development of the Public Accountants, leading healthy lifestyles.
\end{abstract}

Keywords. Certified Public Accountant; Non-Communicable Diseases; Risk Factor's; Healthy habits; Work Environment. 


\section{Introducción}

Este proyecto de investigación se centra en la necesidad de la comunidad de la ciencias contables para adquirir nuevos hábitos y costumbres, teniendo una mejor calidad de vida basada en la práctica de estilos de vida o hábitos saludables, mediante la revisión de modelos que expliquen el impacto que trae consigo la ausencia en la práctica de estos hábitos; resultados que se pueden ver reflejados negativamente en su entorno laboral y social; y resaltando que en Colombia, enfermedades como alcoholismo, tabaquismo y obesidad, entre otras, pueden llegar a causar la muerte, aun cuando se pueden combatir y/o disminuir sus efectos nocivos practicando hábitos que fomente estilos de vida saludables. Es de importancia tener en cuenta que, la vida de un Contador Público genera altos niveles de estrés que pueden afectar de manera negativa su jornada laboral (Loaiza \& Peña, 2013).

En la presente investigación se muestran maneras más efectivas y contundentes para llevar un estilo de vida saludable, tomando en cuenta factores como la poca disponibilidad de tiempo libre del que dispone un Contador Público, buscando su interacción desde el campo o lugar de trabajo apoyándose en herramientas, ejercicios y actividades, de sencilla ejecución y cuyos efectos positivos sustentarán la premisa de la relevancia que trae la implementación estructurada de un manual de instrucciones a seguir.

Fenómenos como la globalización y el avance de una sociedad cada vez más tecnificada, han llevado al sedentarismo, que a su vez, produce en el ser humano problemas de salud, siendo un claro ejemplo, las enfermedades metabólicas que en ocasiones pueden conducir a la muerte.

En consecuencia, es imperativo aplicar un esquema de hábitos saludables dentro del espacio y la jornada laboral del contador que mejoren su calidad de vida, basándose en la ejecución de técnicas fáciles, que requieran poco tiempo de implementación, y que puedan generar paulatinamente, condiciones óptimas de salud (Cigarroa, Sarqui, \& Zapata-Lamana, 2016).

Si Colombia, en toda su dimensión económica, administrativa, contable y financiera, pretende obtener estándares de calidad aplicando normas que generen competitividad en sus empresas, es requisito y necesidad, recurrir a la implementación de hábitos saludables, tal como lo han hecho las grandes potencias mundiales elaboran esquemas de actividades que incluyen eventos físicos, deportivos y de soporte afectivo, son el fin de que sus futuros profesionales manejen 
niveles de energía óptimos, con actitudes asertivas y puedan contribuir al engranaje de su empresa desde las diferentes facetas del ser humano, en un sentido integral (Mendieta \& Salgado, 2013).

Los estilos de vida saludable buscan de manera positiva solucionar problemáticas que van más allá de la salud, y por ende a partir de rutinas que incluyan pequeñas ilustraciones y ejercicios cotidianos, es posible evidenciar que el entorno laboral del contador se ve beneficiado y mejora su calidad de vida. 


\section{Capítulo I. Planteamiento del Problema}

En este capítulo se pretende divulgar la información del trabajo de grado concerniente a la situación problémica, los objetivos y la justificación.

\subsection{Formulación del Problema}

En Colombia, actualmente una de las problemáticas de salud pública que se presenta es la obesidad y el sobrepeso, que afecta al 56\% de la población, sin discriminación entre géneros; y se proyecta que cada 5 años aumentará en un 10\% según una encuesta realizada en el 2015 por la ENSIN, surgiendo de allí la necesidad de disminuir este porcentaje, pues de lo contrario en un par de años alcanzaremos cifras como las de México y Estados Unidos, donde 7 de cada 10 habitantes padecen de obesidad (Fernández \& Suárez, 2017)

En el siglo XXI, las consecuencias de los malos hábitos de vida han aumentado y continúan presentándose con una tendencia ascendente. El campo de la Contaduría Pública no es la excepción. Los Contadores Públicos disponen de poco tiempo para dedicar a actividades y ejercitación, tanto física como deportiva, debido entre otros, al estrés laboral en sobreposición a llevar muy agitadas agendas, lo que trae como consecuencia el padecimiento de Enfermedades Metabólicas No Transmisibles (OMS, 2014), coadyuvadas por cuatro factores de riesgo metabólicos que propenden el riesgo de su padecimiento: hipertensión, hiperglicemia, hiperlipidemia y sobrepeso/ obesidad. Además, se incluyen factores como el desequilibrio hormonal y los patrones no saludables, como el sedentarismo y la alimentación mal planificada (García, Martínez, \& García, 2008).

Es así, como los problemas de salud de etiología metabólica pueden llegar a ser tan perjudiciales y pasar desapercibidos, al punto tal, que el individuo solo las reconoce cuando ya enfermo, sufre sus consecuencias, que en ocasiones tienen efectos devastadores e irreversibles (OMS, 2014).

Entonces, es cuando cobra relevancia el desarrollo del presente trabajo investigativo, mediante el diseño y la implementación de un manual que contenga estrategias prácticas y sencillas de realizar cotidianamente, con el fin de contrarrestar los problemas de salud anteriormente mencionados. 
Cabe señalar, que la mala alimentación es uno de los factores que genera mayores compromisos metabólicos, con efectos colaterales negativos como la disminución de la esperanza de vida promedio.

Dentro del conjunto de parámetros asociados a la mala alimentación, y en el caso específico de los contadores públicos, se encuentran las jornadas laborales, la falta de planificación de pausas para alimentarse, el consumo de alimentos por "oportunidad" y de origen industrial; los cuales originan, a su vez, la disminución de las defensas corporales contra el estrés oxidativo celular, debilitando la barrera frente al efecto nocivo de los radicales libres sobre el ADN, las proteínas y los lípidos corporales, trayendo como consecuencia el aumento indiscriminado en el riesgo de sufrir enfermedades cardiovasculares, degenerativas y cáncer (Cortés, Chiralt, \& Puente, 2005) (Cabezas-Zabala, Hernández-Torres, \& Vargas-Zárate, 2016).

Reflexionando sobre lo anteriormente expuesto y haciendo énfasis en los factores negativos que afectan la salud del Contador, se hace necesaria la implementación de una serie de actividades y recomendaciones dirigidas exclusivamente a mejorar la calidad y hábitos de vida de este grupo de profesionales.

\subsubsection{Pregunta de Investigación.}

¿Cómo contribuir al mejoramiento de la calidad de vida de los Contadores Públicos egresados de la Universidad Santo Tomás de Villavicencio, a partir del uso de estrategias pedagógicas que propendan la implementación de hábitos saludables? 


\subsection{Objetivos}

\subsubsection{Objetivo General.}

Diseñar una estrategia pedagógica (guía) que incluya pautas y lineamientos prácticos para propiciar la realización de actividades diarias tendientes a obtener hábitos saludables en los egresados de Contaduría Pública de la Universidad Santo Tomás sede Villavicencio, permitiéndoles así, mejorar su estilo de vida y ambiente laboral.

\subsubsection{Objetivos Específicos.}

- Caracterizar los hábitos de vida de los Contadores Públicos egresados de la Universidad Santo Tomás sede Villavicencio.

- Analizar distintas variables involucradas en la presencia o no, de hábitos de vida saludable en los Contadores Públicos egresados de la Universidad Santo Tomás Sede Villavicencio.

- Diseñar una estrategia pedagógica con pautas y lineamientos que fomenten la realización de actividades prácticas que ayuden a obtener mejores hábitos saludables en la población a estudio.

\subsection{Justificación}

Comer bien no basta para tener salud. Además, hay que hacer ejercicio, cuyos efectos también deben conocerse. La combinación de ambos factores constituye un régimen. Si hay alguna deficiencia en la alimentación o en el ejercicio, el cuerpo enfermará.

En el mundo mueren 57 millones de personas al año. El aumento de las enfermedades crónicas es el responsable de las dos terceras partes de estas muertes y del $46 \%$ de la morbilidad global, porcentajes que ascienden y proyectan a las enfermedades no transmisibles como causantes del $73 \%$ de la mortalidad y del 60\% de la morbilidad mundial para el año 2020 (OMS, 2014). De los diez factores de riesgo identificados por la Organización Mundial de la Salud (OMS) como 
causantes de las enfermedades crónicas o no transmisibles, cinco guardan estrecha relación con la nutrición y la actividad física: obesidad; sedentarismo; hipertensión arterial; hipercolesterolemia; y consumo insuficiente de frutas y verduras. De tal forma, los dos principales factores (nutrición y actividad física) causantes de las enfermedades crónicas son susceptibles de modificación.

Cabe señalar, que en la sociedad actual, el término salud que se definía como el estado de ausencia de enfermedad, ha sido cambiado insertando en él lo referente a calidad de vida. De tal modo, los sistemas de salud de las sociedades no deben limitar su funcionalidad al no padecimiento de enfermedades, sino que deben apuntar a la promoción y prevención de cuidados que las prevengan. La salud debe dejar de asumirse desde la individualidad del ser, sino como la sumatoria de los efectos que se dan en los individuos que hacen parte de esta, es decir la salud debe ser asumida holísticamente involucrando al sujeto, a la comunidad y su entorno (Cáez-Ramírez \& Casas-Forero, 2007).

Entonces, la promoción de la salud constituye un proceso global político, económico, social y cultural, que envuelve acciones dirigidas a fortalecer las habilidades y capacidades de los individuos, modificando las condiciones ambientales con el fin de mitigar su impacto en la salud pública y personal (Choque, 2005).

En concordancia con lo anteriormente citado, la Organización de Naciones Unidas (ONU) en su Declaración del Milenio (ONU, 2000) promulgó una política sin precedentes, cuyo objetivo general implica el reconocimiento de que "además de las responsabilidades que todos tenemos respecto de nuestras sociedades, nos incumbe la responsabilidad colectiva de respetar y defender los principios de la dignidad humana, la igualdad y la equidad en el plano mundial”.

En Colombia existen 12 millones de personas con obesidad y/o sobrepeso, cerca de un millón tienen diabetes y dos millones de individuos son intolerantes a los carbohidratos. La magnitud de problemas es incalculable. No en vano se puede afirmar, que en la actualidad muere más gente en el mundo como consecuencia de la obesidad que por desnutrición, lo que conduce a pensar acerca de la inequidad en la distribución de la riqueza en el mundo y en la nación, pues Colombia es el segundo país del mundo con mayor desproporción en la repartición de la riqueza, después de Brasil, donde el $80 \%$ del ingreso lo tiene el $20 \%$ de la población. Un equilibrio en los ingresos económicos de la población haría que los ricos no se muriesen por sobrealimentación y los pobres por desnutrición (Escobar \& Rossi, 2007). 
Si las empresas y organizaciones en Colombia se interesaran no solo por el aporte laboral de su empleado en cuanto a la consecución de intereses monetarios o económicos, sino que en contraprestación facilitaran condiciones para mejorar el ámbito laboral, social, espiritual, mental y saludable de los individuos, tendrían muchos más éxitos; tal como lo demuestran industrias de países ubicados en Europa de Norte, donde la mayoría de las empresas confieren a sus empleados mejores estándares laborales, que incluyen la consideración de todos los elementos tendientes a mejorar no solo los procesos productivos, sino también las condiciones, ambiente y clima laboral (Herrero, 2011) .

De allí, que el siguiente trabajo de investigación tiene como fin dar a conocer los diversos resultados que puede encontrar un profesional de la Contaduría Pública al identificar y aplicar dentro de su cotidianidad una serie de actividades prescritas en un manual de ejercicios y pautas para mejorar su calidad de vida, pues generalmente, este grupo poblacional dispone de poco tiempo de relajación y esparcimiento enmarcado dentro de la ejecución de inadecuados hábitos que van en detrimento de su salud. Factores como la nutrición adecuada, una dieta balanceada, una impecable higiene corporal, un asertivo gasto energético y un tiempo suficiente de descanso, sumado a una rutina regular de actividad física promueven el bienestar anatomofisiológico y psicosocial del ser humano, con beneficios tales como el aumento de la autoestima, autoimagen, autoconfianza, disminución del estrés, los estados depresivos y la ansiedad (Velázquez, 2012).

Reconociendo a su vez, que en los últimos tiempos se ha suscitado una cultura del autocuidado para mantener la salud y el bienestar, generando en los consumidores: a) una tendencia mundial al incremento de la demanda de alimentos funcionales, que además de proporcionar nutrientes, aporten un efecto preventivo beneficioso en la salud; b) la creación de espacios de reflexión frente al desafío de promover, más que alimentos seguros, salud, bienestar, longevidad y códigos de conducta; c) un genuino interés por conocer cómo integrar las nuevas concepciones de salud y alimentación, dentro de un contexto ético acorde con el paradigma de la persona como totalidad; y d) una conciencia sobre los preceptos culturales que deben ajustarse por ser el resultado, en gran medida, de la pobreza, la ignorancia, los prejuicios y la escasez de alimentos (Cáez-Ramírez \& Casas-Forero, 2007)

Es relevante recalcar que, a los preceptos ampliamente establecidos con anterioridad, se debe sumar el de un entorno saludable; siendo este definido como el espacio físico, social y cultural donde de manera cotidiana se cohabita, ya sea su fin el de vivienda, educación, lugar de trabajo, 
municipio o país, entre otros, en el cual se establecen relaciones sociales en condiciones básicas de saneamiento ambiental, logrando la generación de ámbitos psicosociales sanos y seguros en armonía y equilibrio (OPS, 2016).

En el caso de los Contadores Públicos, se hace necesario tratar de transformar los espacios dedicados a las jornadas laborales con el fin de generar entornos saludables que propendan por el bienestar de quienes conviven en él. De manera consecuente, es importante llevar a cabo la presente investigación, para facilitar la creación de una cultura de hábitos saludables que estimulen la generación de aportes positivos en la vida de los Contadores Públicos, dando sustancialmente un punto de equilibrio entre la salud del Contador Público y el desempeño laboral que pueda tener al mejorar sus condiciones de vida. Por ello, se plantea un manual en el que se oriente la forma de dejar atrás las desatinadas conductas y que evidencie los progresos que logra el individuo al adoptar hábitos saludables desde múltiples perspectivas y facetas. 


\section{Capítulo II. Bases Teóricas}

En este apartado, se incluye la revisión de antecedentes desde tres aspectos básicos: conceptuales, investigativos y legales.

\subsection{Marco Conceptual}

En el año 2015, se llevó a cabo la Encuesta Nacional de la Situación Nutricional (ENSIN 2015), que entre otros, mostró como resultados que en Colombia, la población con rango etario entre 18 y 64 años cuenta con un $56.4 \%$ de personas afectadas por algún grado de exceso de peso, que en el caso del género femenino abarca un total del 59.5\% y en los hombres 52.7\%, reportando así una obesidad del 37.7\% (MinSalud, 2017).

\subsubsection{Hábitos de Vida Saludable.}

Los hábitos de vida saludable se entienden como el equilibrio logrado a partir de una intrincada relación entre el bienestar físico y mental (OMS, 2018), que incluye patrones de consumo individuales como la nutrición, el tabaquismo y la actividad física, entre otros; y ante los cuales se deben asumir cuatro compromisos programáticos como factores de protección que son comer sano, disfrutar de la familia, vivir al aire libre y mover el cuerpo (DeÀguila, 2012). Esto se logra, a través de la implementación de pautas cotidianas sencillas, tales como iniciar la jornada con un desayuno, almuerzo moderado y cena liviana (Casado-Pèrez, Hernàndez-Barrera, CarrascoGarrido, \& Palacios-Ceña, 2015). En palabras de Oblitas (2008):

La promoción de estilos de vida saludables implica conocer aquellos comportamientos que mejoran o socavan la salud de los individuos. Se entiende por conductas saludables aquellas acciones realizadas por un sujeto, que influyen en la probabilidad de obtener consecuencias físicas y fisiológicas inmediatas y a largo plazo, que repercuten en su bienestar físico y en su longevidad (p. 226).

De tal modo, que para lograr tener hábitos de vida saludable es requisito adoptar comportamientos rutinarios concretos que contemplen, entre otros, el no consumo de sustancias 
nocivas, pues estas se asocian al riesgo de sufrir enfermedades cerebrovasculares, respiratorias y hasta cáncer (Schukitt, 1995).

Es de señalar que la formulación de acciones que conlleven a la obtención de hábitos de vida saludable, debe estar enmarcado dentro de estrategias integrales y transversales que privilegien la modificación de comportamientos individuales, familiares y comunitarios, a partir de la acción conjunta entre el aumento de la actividad física y el establecimiento de pautas alimentarias asertivas (Jalisco, 2012).

Es decir, los estilos de vida pueden ser definidos como las manifestaciones de dimensiones colectivas y sociales, que a su vez son determinados y modelados por el contexto social, económico

y cultural, y que deben ser discutidos desde perspectivas como la de desarrollo biopsicosocial y la salud humana (Pedersen, Huayllasco, \& Erràzuriz, 2007)

En cuanto al desarrollo de hábitos de vida saludables, es requisito considerar la perspectiva del enfoque sociocultural, en el cual el hombre como ser biológico, vive en un grupo humano específico y es creador y portador de cultura, ya que el establecimiento de estos hábitos se ve permeado por tendencias poblacionales como la conceptualización particular de dicha comunidad, cómo estas mantienen o luchan por alcanzar su logro y cuáles son sus condicionantes (Pedersen D. , 1998).

En el caso específico de los Contadores Públicos, al realizar la mayor parte de su desempeño profesional al interior de una oficina y pasando mucho tiempo sentados durante su horario laboral, es acertado señalar que la construcción de hábitos de vida saludable debe incluir parámetros como las pausas activas, evitar tener la misma posición estática por tiempo prolongado, cambiar continuamente la postura, alimentarse saludablemente, evitar el consumo de alcohol y el tabaquismo, al igual que buscar métodos para aliviar el estrés, entre otros (Buckley et al., 2015)

\subsubsection{Enfermedades Crónicas o No Transmisibles (ENT).}

Las ENT, también conocidas como enfermedades crónicas, tienden a ser de larga duración y resultan de la combinación de factores genéticos, fisiológicos, ambientales y conductuales. Los principales tipos de ENT son las enfermedades cardiovasculares (como los ataques cardiacos y los accidentes cerebrovasculares), el cáncer, las enfermedades respiratorias crónicas (como la enfermedad pulmonar obstructiva crónica -EPOC- y el asma) y la diabetes (OMS, 2014). 
Las enfermedades cardiovasculares generan la muerte de más de 17 millones de personas en el mundo, en segundo lugar, el cáncer ocasionando la muerte de 9 millones de personas en el mundo, después y en tercer lugar las enfermedades relacionadas con el sistema respiratorio con 3.9 millones de personas muertas cada año y por último la diabetes con más de 1.6 millones de personas muertas cada año (OMS, 2014).

En términos generales, se puede afirmar que las ENT se desarrollan por la carencia de hábitos de vida saludable sumados al manejo de altos niveles de estrés, que pueden causar no solo el deterioro de la calidad de vida, sino el padecimiento de secuelas físicas, mentales y cognitivas, y la muerte.

\subsubsection{Obesidad.}

La obesidad se define como un aumento del tejido adiposo corporal. Esta acumulación de grasa se traduce en un incremento del peso y aunque no todo incremento del peso corporal es debido a un aumento del tejido adiposo generando complicaciones en la salud del ser humano, en la práctica médica el concepto de obesidad está relacionado con el peso corporal (Pèrez, 2014). La obesidad es una ENT que se desarrolla debido a una conjunción de elementos multifactoriales de origen etiológico diverso, que trae consigo el detrimento de la salud física y mental, pues su padecimiento afecta varias dimensiones humanas, la autoestima y la autoimagen.

Cifras obtenidas de ENSIN - 2015 (MinSalud, 2017) revelan que el 56.4\% de los colombianos en edades entre 18 y 64 años tiene sobrepeso o padece de obesidad cifra que aumentó en $5.2 \%$ en comparación al año 2010, donde fue de 51.2\%, siendo en ambos casos, el género femenino en quienes se presenta un mayor grado. Tal como lo afirma (Cáez-Ramírez \& CasasForero, 2007):

La obesidad, como un tema de salud pública de primera prioridad en el mundo entero, tiene sus raíces en un cambio de comportamiento, que no es solo responsabilidad personal, sino que está influido por numerosas variables ambientales en el área de las relaciones económicas, de las dinámicas culturales y de los modelos de vida y de satisfacción de necesidades, todas las cuales afectan los patrones de alimentación y de actividad física de una población. A partir de esta aseveración, un enfoque exitoso debe incorporar una mirada integral y transversal del problema (p. $105)$. 


\subsubsection{Hipertensión Arterial.}

Es la elevación de los niveles de presión arterial de forma continua o sostenida. Para entenderlo mejor es importante definir la presión arterial: El corazón ejerce presión sobre las arterias para que éstas conduzcan la sangre hacia los diferentes órganos del cuerpo humano. Esta acción es lo que se conoce como presión arterial. La presión máxima se obtiene en cada contracción del corazón y la mínima, con cada relajación. (FunEsCo, 2018). Esto es, que la presión arterial como comportamiento corporal, hace que la sangre fluye rápidamente por las arterias ocasionando que la frecuencia cardiaca se acelere, y si esta no es controlada a tiempo, puede llevar a problemas de mayor gravedad como ataques cardiacos o los accidentes cerebrovasculares $(\mathrm{ACV})$.

Cabe resaltar que uno de los problemas de salud pública que afecta al planeta entero, es la hipertensión arterial, pues 4 de cada 10 personas la padece, siendo la población mayor de 50 años quienes tienen mayor riesgo de sufrirla. En Colombia, durante el año 2015, se reportó que 7 de cada 10 personas entre 18 y 69 años presentan problemas de hipertensión arterial (MinSalud, 2017).

\subsubsection{Diabetes.}

La diabetes es una enfermedad crónica que aparece cuando el páncreas no produce insulina suficiente o cuando el organismo no utiliza eficazmente la insulina que produce. (OMS, 2017). La diabetes puede ser de tipo 1, tipo 2 o gestacional. La diabetes de tipo 1, denominada también Insulinodependiente, juvenil o de inicio en la infancia, se caracteriza por una producción deficiente de insulina y requiere la administración diaria de esta hormona. Se desconoce aún la causa que la origina y no se puede prevenir con el conocimiento actual.

Por su parte, la diabetes tipo 2, denominada No Insulinodependiente o de inicio en la edad adulta, se debe a una utilización ineficaz de la insulina por parte del organismo. Este tipo representa la mayoría de los casos mundiales y se debe en gran medida a un peso corporal excesivo y a la inactividad física.

Mientras que la Diabetes Gestacional se caracteriza por hiperglucemia que aparece durante el embarazo y alcanza valores que, pese a ser superiores a los normales, son inferiores a los establecidos para diagnosticar una diabetes. Las mujeres con diabetes gestacional corren mayor 
riesgo de sufrir complicaciones durante el embarazo y el parto. Además, tanto ellas como sus hijos corren mayor riesgo de padecer diabetes de tipo 2 en el futuro (OMS, 2017).

En Colombia, la Diabetes es un problema de salud pública, pues más de 3.3 millones de personas la sufren, de las cuales solo 2.2 están diagnosticadas y más de un millón no saben que la padecen porque aún no han sido diagnosticadas (Caracol Radio, 2016)

\subsubsection{Sedentarismo.}

El sedentarismo se define como la falta de realizar actividad física durante al menos 15 minutos tres veces por semana, durante un trimestre; mientras la actividad física hace referencia a cualquier movimiento del cuerpo producido por los músculos esqueléticos que resulte en pérdida de energía (Álvarez F. , 2010).

La falta de actividad física es un riesgo para la salud mundial, es un problema extendido y en rápido ascenso en países, tanto desarrollados como en vías de desarrollo; así que para lograr mejores resultados en la prevención de las enfermedades crónicas, es muy importante que las estrategias y las políticas que se apliquen tengan en cuenta el papel fundamental de la actividad física como marcador de riesgo para la ocurrencia de las mismas.

Según la Organización Mundial de la Salud más del 60\% de la población mundial no realiza rutinas que incluyan una mínima actividad física, fomentando así la generación de problemas de salud como ENT (OMS, 2018).

Esto indica, que debido a una serie de determinantes sociodemográficos, individuales y familiares, una gran proporción de personas en el ámbito mundial, se encuentran en riego de padecer algún tipo de enfermedad crónica, como resultado de la disminución del gasto de energía (y consecuentemente, aumento en la grasa corporal), producto de comportamientos sedentarios y falta de actividad física (Dietz, 1998).

Colombia presentan una panorama negativo en sus niveles de actividad física, según un informa realizado por la OMS en el año 2015 Colombia es un país donde más del 60\% de sus habitantes no alcanza niveles mínimos de actividad física, lo que conlleva posiblemente a un deterioro de la calidad de vida, ya que la relación baja actividad física/ ocurrencia de ENT ha sido ampliamente probada (Vázquez, 2016) 


\subsubsection{Ataque Cardiaco (Infarto Miocárdico).}

Un ataque cardíaco ocurre cuando se obstruye de repente el flujo de sangre que llega a una parte del corazón y este no puede obtener oxígeno que requiere para el funcionamiento corporal. Si el flujo de sangre no se restablece rápidamente, esa parte del corazón se deteriora al punto de morir sus células (HHS, 2018).

En Colombia, las enfermedades coronarias encabezadas por el ataque cardiaco determinan el $16.9 \%$ del total de muertes anuales. Factores de riesgo cardiovascular, ya sean biológicos o conductuales, que han sido establecidos son: el tabaquismo, la hipercolesterolemia, la diabetes, la hipertensión arterial, la obesidad, la falta de ejercicio físico regular y/o el sedentarismo, los antecedentes familiares y el estrés. Además de fatores específicos en las mujeres, como ovarios poliquísticos, anticoncepción oral y producción de estrógenos. Cuanto mayor sea el nivel de cada factor de riesgo, mayor es el riesgo de tener una enfermedad cardiovascular (Sans-Menéndez, 2007).

\subsubsection{Nutrición: Alimentación Saludable y Balanceada.}

La nutrición es la ingesta de alimentos en relación con las necesidades dietéticas del organismo. Una buena nutrición (una dieta suficiente y equilibrada combinada con el ejercicio físico regular) es un elemento fundamental de la buena salud. Una mala nutrición puede reducir la inmunidad, aumentar la vulnerabilidad a las enfermedades, alterar el desarrollo físico y mental, y reducir la productividad (OMS, 2018).

Básicamente, dentro de un buen régimen nutricional, se recomienda a cada persona comer al menos 5 porciones de frutas y verduras, o en su defecto 400 gramos/día, ya que de esta esta manera se logra reducir el riesgo de desarrollar ENT y se proporciona una ingesta balanceada de fibra al organismo; también se recomienda un consumo moderado de sal diario entre 3 y 5 gramos/día para no desarrollar problemas de hipertensión arterial. En cuanto al azúcar, se recomienda no consumir bebidas azucaradas (como refrescos, gaseosas y demás) o productos con

altas cantidades de azúcar refinada, ya que su consumo constituye un factor de riesgo para la ocurrencia de la diabetes (OMS, 2018).

Una alimentación balanceada incluye el consumo adecuado de micro y macronutrientes que necesita una persona para llevar una vida saludable. Los micronutrientes hacen referencia a las vitaminas y minerales necesarios para evitar enfermedades causadas por infecciones bacteriales 
o víricas. Los macronutrientes son las proteínas, carbohidratos y grasas de origen vegetal que proporcionan energía para las actividades diarias.

De otra parte, el consumo de agua es un factor que no se debe dejar sin mencionar. El agua esta presente en el $70-85 \%$ del organismo, siendo su principal componente; de este porcentaje, se cree que alrededor del 65 - 80\% se encuentra intracelularmente y el resto, puede hallarse circulando en sangre o alojada en tejidos.

De allí la importancia de hidratarse correctamente para lograr hábitos de vida saludables, pues la hidratación constante puede ser considerada como un factor protector. Por ende, se recomienda que el consumo de agua debe ser alrededor de 30 - $35 \mathrm{ml} / \mathrm{kg}$ de peso, teniendo en consideración que los alimentos, como frutas y verduras, sólo aportan el 20\% del agua requerida, mientras que el $80 \%$ se obtiene del consumo de agua pura líquida (Albertman, 2017)

\subsubsection{La Importancia del Sueño.}

El sueño es un fenómeno complejo resultante de la interacción entre el funcionamiento del sistema neuroendocrino, los relojes biológicos y procesos bioquímicos, con aspectos ambientales, culturales y sociales que adquieren especial relevancia en cada etapa de la vida. La función reparadora del sueño y las consecuencias que su alteración produce en las funciones cognoscitivas, el desempeño diurno y la salud en general, son algunos de los elementos estudiados por las neurociencias (Talero-Gutiérrez, Durán-Torres, \& Pérez-Olmos, 2013).

En el mundo de la neurociencia, existen muchos interrogantes sobre cuánto tiempo un ser humano debe dormir, lo que si se ha establecido sólidamente es la importancia de éste, ya que el cuerpo y el cerebro deben tener espacios en el cual alcancen estados de relajación donde las pulsaciones cardiacas disminuyan y la presión sanguínea se regule.

Es de anotar, que un buen consumo de alimentos sumado a unas adecuadas horas de sueño, en adultos que laboran entre 7 - 9 hr/día, bajo elevadas condiciones de estrés, hace que el cuerpo se autorregule y pueda recuperarse de su desgaste diario (Manes, 2015).

La investigación sobre la biología del sueño y sobre los efectos clínicos de sus trastornos cada vez deja más en claro que el sueño es un proceso de vital importancia para la salud integral del ser humano. Sin embargo, según algunos estudios, la tendencia en la población mundial es hacía la reducción del tiempo total de sueño, lo cual se ha reflejado en el incremento en la incidencia de trastornos del sueño (Carrillo-Mora, Ramírez-Peris, \& Magaña-Vázquez, 2103). 


\subsubsection{Las Pausas Activas.}

Las pausas activas son sesiones de actividad física desarrolladas en el entorno laboral, con una duración continua mínima de 10 minutos que incluye la adaptación física cardiovascular, el fortalecimiento muscular y el mejoramiento de la flexibilidad, que buscan reducir el riesgo cardiovascular y las lesiones musculares por sobreuso asociados al desempeño laboral.

Las Pausas Activas son una forma de promover la actividad física, como habito de vida saludable, con los beneficios propios de esta.

En el entorno laboral, y en el caso de los Contadores Públicos, la gran mayoría permanecen por lo menos ocho horas del día y generalmente, están sentados, con un nivel de actividad física mínimo.

Esto favorece que las personas pierdan su condición física, lo cual a su vez promueve una actitud sedentaria relacionada directamente con cambios metabólicos y estructurales en el cuerpo humano. así que el contenido de las pausas activas debe estar encaminado a mejorar la condición física de los trabajadores.

Por lo tanto, deben considerar el trabajo de las capacidades físicas, al mejorar la condición física de los trabajadores, se tenderá a disminuir el sedentarismo como base fundamental de numerosas ENT y se podrá disminuir el riesgo de aparición de patologías relacionadas con el sobrepeso, el sistema cardiovascular y el osteomuscular, entre otros (MinSalud, 2015).

\subsubsection{Estrategias Pedagógicas.}

Las estrategias pedagógicas conciernen un proceso organizado, formalizado y orientado que busca la obtención de una meta establecida; su concepto involucra la selección de actividades y prácticas en diferentes momentos formativos, métodos y recursos; guía la acción y el sentido que orienta la obtención de resultados, da sentido y coordinación a todo lo que se hace para llegar a la meta.

Es decir, las estrategias pedagógicas son un conjunto de procedimientos, apoyados en técnicas de enseñanza, que tienen por objeto llevar a buen término una acción pedagógica, apuntan a fomentar procesos de autoaprendizaje, aprendizaje interactivo y aprendizaje colaborativo; y para su definición es fundamental tener clara la disposición del grupo al que se va a aplicar, su edad y contexto (UDistrital, 2012). 
Quien oriente la estrategia pedagógica debe seleccionar de manera consciente, reflexiva y crítica las técnicas y actividades que permitan alcanzar los objetivos propuestos, teniendo en cuenta varios componentes, entre otros, la edad del grupo, su disposición para aprender, las características sociales de los mismos, su desarrollo cognitivo y afectivo, y los recursos necesarios para llevar a cabo esta actividad, fomentando el autoaprendizaje, el aprendizaje interactivo y el aprendizaje cooperativo (Velasco \& Mosquera, 2007).

\subsection{Antecedentes Investigativos}

En investigación denominada "Promoción de estilos de vida saludable en el Área de salud de Esparza", las autoras Acuña \&Cortes (2012) plantearon como objetivo el promover estilos de vida saludable en la población adscrita al Área de Salud de Esparza, Costa Rica; encontrando que respecto a los hábitos de vida saludable, la población a estudio, con edades entre los 20 - 49 años, es algo sedentaria, pues solo realizan actividad física 1 o 2 veces por semana (Acuña \& Cortes, 2012).

De otra parte, en estudio realizado por Grimaldo (2010) llamado "Calidad de vida y estilo de vida saludable en un grupo de estudiantes de pregrado de la ciudad de Lima" se planteó como objetivo determinar la relación entre los dominios de bienestar económico, amigos, vecindario y comunidad, pareja, vida familiar, ocio, medios de comunicación, religión y calidad de vida y tres factores del estilo de vida saludable (actividad deportiva, consumo de alimentos y sueño y reposo) en estudiantes de posgrado de Ciencias de la Salud de la ciudad de Lima, Perú.

Dentro de los resultados encontrados, se obtuvo que existe una estrecha relación entre sueño y calidad de vida; también se evidencio correlación entre calidad de vida del dominio salud y el consumo de alimentos saludables; y se observaron correlaciones moderadas entre algunos dominios de calidad de vida (amigos, vida familiar, ocio y religión) con el sueño y el reposo; a pesar de no encontrar correlación entre calidad de vida y actividad deportiva (Grimaldo, 2010).

En la investigación "Promoción de salud y estilos de vida saludables en jóvenes universitarios: Hacia un curriculum integral", realizada por Rojas \& Siegel (2011) en la Universidad Austral de Chile, se plantea como objetivo de estudio evaluar la presencia de fatores de riesgo cardiovascular, determinando los estilos de vida y promoviendo la adopción de factores protectores para la salud de los estudiantes a través de un programa de intervención de cinco meses. 
El procedimiento incluyó el registro de componentes biomédicos como el Índice de Masa Corporal (IMC), perímetro de cintura, perfil lipídico, glicemia y una encuesta autoaplicada de estilos de vida, para luego desarrollar sesiones enfocadas en la promoción de la salud, la actividad recreativa, la ejercitación física, la alimentación saludable, la psicoeducación, la disminución en el consumo de tabaco y alcohol.

Dentro de los resultados se obtuvo que la población a estudio presenta estilos de vida poco saludables, destacando entre otros, los elevado índices de sobrepeso, obesidad y sedentarismo, los cuales a largo plazo pueden conducir al desarrollo de ENT, como las cardiovasculares y el cáncer (Rosas \& Siegel, 2011).

En estudio realizado por Reyes (2008) y nombrado "Factores que intervienen a llevar estilos de vida saludables en el personal de Enfermería de los servicios de cirugía y medicina del Hospital Nacional de San Benito, Petén", la autora señala como objetivo describir los factores que intervienen en el personal de enfermería para llevar estilos de vida saludables y obtuvo como resultado que el $61 \%$ del personal no tiene claro los conceptos y las prácticas de buenos hábitos de salud, al igual que se determinó que es necesaria la participación del hospital para generar o propender prácticas de estilos de vida saludables (Reyes, 2008)

Bennasar-Veny (2012) publica su investigación "Estilos de vida y salud en estudiantes universitarios: La universidad como entorno promotor de la salud", donde pretende conocer y analizar estilos de vida del alumnado de la Universitat de les Illes Balears.

Para ello, diseñó y validó un instrumento que evalúa los estilos de vida y el estado de salud de los universitarios (ESVISAUN). Entre los resultados obtenidos, destaca que el cuestionario ESVISAUN puede considerarse un instrumento válido y fiable para identificar hábitos de vida saludables y problemas de salud con el objetivo de diseñar intervenciones en el marco de la promoción de la salud en el entorno universitario.

También se obtuvo que los resultados reafirman la idea de que los comportamientos de salud de los universitarios no deben de considerarse de forma aislada unos de otros, ya que se observa una tendencia a la conglomeración de factores de riesgo como fumar, dieta no saludable e inactividad física (Bennasar-Veny, 2012).

El estudio denominado "Estilos de vida saludable en los estudiantes internos de enfermería de la Universidad de Guayaquil 2014 - 2015”, Álvarez \& Andaluz (2015) proponen como objetivo 
principal determinar los estilos de vida saludables en la población señalada, tomando como contexto el desarrollo de sus actividades académicas y laborales.

Luego de la aplicación de sus instrumentos de recolección de información, señalaron como conclusiones que tienen el $50 \%$ consume de 2 a 3 porciones verduras al día; un $60 \%$ consume de 2 - 4 porciones de frutas, pero que también el 50\% consume comida chatarra como dulces, tortas y frituras. En cuanto a la actividad física, la que más realizan son las caminatas teniendo una participación de un 70\% de los estudiantes internos (Álvarez \& Andaluz, 2015).

Daza \& Ramos (2014) publican su investigación "Estilos de vida saludable de los estudiantes de secundaria del Instituto Colombo - venezolano de la ciudad de Medellín”, en la cual a partir del corte empírico analítico y la aplicación de instrumentos convalidados.

Se obtuvo que: a) existe una correlación inversamente proporcional entre el grado de escolaridad y los estilos de vida saludable; b) el nivel de escolaridad de los padres y los estilos de vida saludable tienen una relación directamente proporcional; c) la orientación religiosa incide en los comportamientos de vida saludable, toda vez que los estudiantes sin ninguna orientación religiosa demostraron tener menos hábitos saludables, en tanto los estudiantes con orientación religiosa adventista, mostraron mejores resultados; d) los estudiantes de sexo masculino superan a las mujeres en la práctica de actividad física; e) el estrato socioeconómico de los padres tiene una relación directamente proporcional con los hábitos de vida saludable de los estudiantes (Daza \& Ramos, 2014).

En trabajo investigativo llevado a cabo por Quintero (2014) y nombrado "Hábitos alimentarios, estilos de vida saludables y actividad física en neojaverianos del programa nutrición y dietética de la PUJ - Primer período 2014”, de corte observacional descriptivo, se planteó como objetivo identificar los hábitos alimentarios, los estilos de vida saludables y el nivel de actividad física en la población nombrada; evaluándose parámetros como nutrición balanceada, tabaquismo, consumo de bebidas alcohólicas, sustancias psicoactivas y bebidas energizantes, y la práctica moderada de actividad física.

Se obtuvo como resultado que el $10 \%$ de la población a estudio tiene sobrepeso, $13 \%$ son fumadores, $37 \%$ consumen bebidas alcohólicas, 50\% practican actividad física moderada y 37\% manejan un nivel de actividad física alto (Quintero, 2014). 


\subsection{Marco Legal}

Para el desarrollo de una buena práctica de los hábitos y estilos de vida saludable la Constitución Política de Colombia (Senado, 1991) regula en los siguientes artículos para una sociedad con mejores niveles de vida y salud los siguientes

- Artículo 49. La atención de la salud y el saneamiento ambiental son servicios públicos a cargo del Estado. Se garantiza a todas las personas el acceso a los servicios de promoción, protección y recuperación de la salud. Corresponde al Estado organizar, dirigir y reglamentar la prestación de servicios de salud a los habitantes y de saneamiento ambiental conforme a los principios de eficiencia, universalidad y solidaridad. También, establecer las políticas para la prestación de servicios de salud por entidades privadas, y ejercer su vigilancia y control. Así mismo, establecer las competencias de la Nación, las entidades territoriales y los particulares, y determinar los aportes a su cargo en los términos y condiciones señalados en la ley. Los servicios de salud se organizarán en forma descentralizada, por niveles de atención y con participación de la comunidad. La ley señalará los términos en los cuales la atención básica para todos los habitantes será gratuita y obligatoria. Toda persona tiene el deber de procurar el cuidado integral de su salud y la de su comunidad.

- Artículo 52. El ejercicio del deporte, sus manifestaciones recreativas, competitivas y autóctonas tienen como función la formación integral de las personas, preservar y desarrollar una mejor salud en el ser humano. El deporte y la recreación, forman parte de la educación y constituyen gasto público social. Se reconoce el derecho de todas las personas a la recreación, a la práctica del deporte y al aprovechamiento del tiempo libre. El Estado fomentará estas actividades e inspeccionará, vigilará y controlará las organizaciones deportivas y recreativas cuya estructura y propiedad deberán ser democráticas.

- Artículo 79. Todas las personas tienen derecho a gozar de un ambiente sano. La ley garantizará la participación de la comunidad en las decisiones que puedan afectarlo. Es deber del Estado proteger la diversidad e integridad del ambiente, conservar las áreas de especial importancia ecológica y fomentar la educación para el logro de estos fines. 
Además, desde el Congreso de la República se han creado leyes que decretan la búsqueda de mejoras en la calidad de vida de los colombianos, con el fin de comenzar a crear conciencia, no sólo en los individuos, sino también en las empresas y la comunidad. Algunos de estos lineamientos son:

- Ley 1355 del 14 de octubre del 2009. En la cual define a la obesidad y las enfermedades crónicas no transmisibles asociadas a esta como una prioridad de salud pública y se adoptan medidas para su control atención y prevención (MEN, 2009).

- Decreto 2771 del 2008. Por el cual se crea la Comisión Nacional Intersectorial para la coordinación y orientación superior del fomento, desarrollo y medición de impacto de la actividad física (COLDEPORTES, 2008).

- Ley 1122 del 2007. Donde constituye en sus artículos 32 y 33 que la salud pública es de vital importancia y que la práctica de actividad física tanto colectiva o individual ayuda a identificar las condiciones de vida bienestar y desarrollo del país (MinSalud, 2007).

Cabe señalar que a partir de la Constitución Política de 1991, en Colombia la salud se convierte en un derecho y en un servicio público a cargo del Estado. Para lograr este objetivo se debe garantizar a todas las personas el acceso a los servicios de promoción, prevención, protección y recuperación por medio del aseguramiento universal.

Con la Ley 100, las EPS, ARS y otras entidades asociadas al Sistema Nacional de Salud han hecho esfuerzos no articulados y poco efectivos en temas relativos a la promoción y prevención en salud pública (Cáez-Ramírez \& Casas-Forero, 2007).

Para garantizar que cualquier sistema de salud funcione, este debe contar con cinco características esenciales: accesibilidad, oportunidad, seguridad, pertinencia y continuidad. Esta normatividad, la posterior y su ejecución carecen de un contenido y actuaciones acordes con dichos postulados, lo cual ha fomentado nuevos proyectos de ley que la reformulen para hacerla más viable y equitativa (Cuéllar-Montoya, 2005); sin contar con un sistema eficiente, que garantice la atención primaria en salud, es limitado el espectro de estrategias que se pueden esperar en relación con diagnósticos y acciones precisas. 


\section{Capítulo III. Metodología}

\subsection{Enfoque, Alcance y Diseño}

Para el desarrollo del trabajo, se orienta la investigación bajo el enfoque mixto, es decir cualitativo y cuantitativo, ya que a partir de la recolección de información por medio de una encuesta se proyecta determinar los estilos de vida saludables o no, de los profesionales de Contaduría Pública de la Universidad Santo Tomas en Villavicencio.

La investigación tiene un fin aplicable ya que pretende plantear una estrategia pedagógica (guía), sencilla y fácil de comprender y ejecutar, que explique la importancia de incentivar el consumo de alimentos sanos, dentro de una alimentación saludable y balanceada; y la pertinencia en la realización de actividad física, según sus distintas variantes y contextos, señalándole al Contador Público la necesidad de llevar hábitos de vida saludable. Esta propuesta evidencia la importancia de llevar a cabo hábitos de vida saludable de manera continua y constante, creando así una cultura de autocuidado, en el ambiente tanto laboral como personal.

La investigación tiene contiene dentro de su marco estructural fases exploratorias que nos permitan evidenciar cuales son los estilos de vida que tiene cada Contador Público y como desde los Hábitos de Vida Saludable se pueden mejorar. También trae consigo una propuesta pedagógica con el fin de que la vida de los Profesionales del Área Contable mejore y se evidencia su impacto no solo en la sociedad contable, sino en el ámbito de la salud disminuyendo los riesgos de sufrir ENT.

\subsection{Hipótesis}

A partir de la formulación de la hipótesis, el investigador anticipa una explicación probable de los fenómenos o del hecho que se estudia y plantea las respuestas al mismo. Tal como lo afirma Monje (2011):

La hipótesis predice el resultado esperado y establece las relaciones que el investigador espera encontrar como resultado del estudio (...) se indican las expectativas del investigador con respecto a las relaciones entre las variables a estudio. Las hipótesis son entonces suposiciones que permiten al 
investigador postular relaciones entre los fenómenos bajo investigación, prediciendo cómo se relacionan dichos fenómenos (...) proporciona guía y orientación a la investigación y un enfoque más formal para la recolección e interpretación de datos; luego de su comprobación se incorpora a la teoría generando nuevo conocimiento (p. 24).

Teniendo en cuenta las clases de hipótesis que pueden tener las investigaciones, según diversos parámetros, se determina que para el presente trabajo se contará con una hipótesis investigativa (Hernández-Sampieri, Fernández, \& Baptista, 2014).

\section{- Hipótesis Investigativa (Hi).}

Al caracterizar los hábitos de vida de los Contadores Públicos se pueden diseñar estrategias pedagógicas que incluyan pautas y lineamientos prácticos que propicien la realización de actividades diarias, mejorando así su estilo de vida y ambiente laboral.

\subsection{Variables}

En investigación, una variable es una propiedad que puede fluctuar y cuya variación es susceptible de medirse u observarse. Este concepto se aplica a personas, seres vivos, objetos, hechos y fenómenos, los cuales adquieren diversos valores respecto de la variable referida. Las variables adquieren valor para la investigación cuando se relacionan con otras variables, es decir, si forman parte de una hipótesis o una teoría (Hernández et al., 2014).

En el presente trabajo se consideran como variables las que aparecen señaladas en la tabla 1.

Tabla 1. Variables Investigativas

\begin{tabular}{|l|l|l|}
\hline Variable Independiente & Variable Dependiente & \multicolumn{1}{|c|}{ Operativización de la Variable } \\
\hline \multirow{4}{*}{ Hábitos de Vida Saludable } & \multirow{3}{*}{$\begin{array}{c}\text { Enfermedades No } \\
\text { transmisibles }\end{array}$} & Padecimiento de Diabetes diferente a la Gestacional \\
\cline { 3 - 3 } & & Padecimiento de Hipertensión Arterial \\
\cline { 3 - 3 } & \multirow{4}{*}{ Hábitos Alimenticios } & Padecimiento de Hiperlipidemia \\
\cline { 2 - 3 } & & Consumo de frutas \\
\cline { 2 - 3 } & & Consumo de verduras \\
\cline { 2 - 3 } & Hábitos Higiénicos & Lavado de manos \\
\cline { 2 - 3 } & Actividad Física & Pausas activas realizadas \\
\cline { 2 - 3 } & Bienestar Emocional & Tiempo en familia \\
\cline { 2 - 3 } & Descanso & Horas de sueño \\
\hline
\end{tabular}

NOTA: Descripción de los habitos de vida saludable, por Daniel Andrés Garzón Céspedes, 2019 


\subsection{Población y Muestra}

En la presente propuesta investigativa, la población o universo de estudio está conformada por egresados de Contaduría Pública de la Universidad Santo Tomás, sede Villavicencio, de los últimos dos años, a quienes se les aplica la técnica de muestreo discrecional para seleccionar a los individuos participantes.

El muestreo discrecional es una técnica de muestreo no probabilístico, donde el investigador selecciona las unidades de observación que sirven como muestra, basándose en su conocimiento y juicio profesional, seleccionando una muestra más representativa que pueda arrojar resultados más precisos (Explorable, 2018). Esta muestra incluye a un total de 68 egresados.

\subsection{Técnicas e Instrumento de Recolección de Datos}

Se diseña un cuestionario, avalado por el Dr. Orlando Bermúdez, Médico de la Universidad del Norte, y Deportólogo de la Fundación Universitaria de Ciencias de la Salud, que incluye hábitos y estilos de vida saludable en un total de 9 preguntas. De tal modo que 4 preguntas hacen referencia al tema de higiene y salud; 3 preguntas al tema de alimentación; 1 pregunta en el tema de ejercicio; y 1 pregunta al bienestar emocional (Apéndice B).

Es importante decir que en el momento de la elaboración de la encuesta se tiene en cuenta una serie de respuestas abiertas donde hay respuestas positivas y negativas encaminadas a determinar la calidad de vida del encuestado.

\subsection{Procedimiento}

La presente investigación se llevó a cabo en tres fases que se describen a continuación.

- Fase 1. Se realiza una revisión exploratoria, que permite rastrear y recopilar información sobre los hábitos de vida saludable del Contador Público, y se constituye en la base para diseñar el instrumento de recolección de información (encuesta) que permita caracterizar los hábitos a la hora de llevar una vida saludable. 
- Fase 2. Aplicar la encuesta y a partir de la recolección de datos, analizar la información que permita describir los hábitos de vida, saludables o no, que tiene la población objeto de estudio, desde dimensiones como la social, emocional, individual y cultural.

- Fase 3. Diseñar una estrategia pedagógica, basada en la elaboración de una guía de fácil compresión, que promueva los hábitos de vida saludable y sus beneficios para los Contadores Públicos; y que incluya pautas relacionadas con la nutrición balanceada, la actividad física, el tiempo de descanso y las pausas activas, entre otros, con elementos específicos para este grupo poblacional. 


\section{Capítulo IV. Análisis e Interpretación de Datos}

A partir de lo instrumentos aplicados, se obtiene información referente al objeto de estudio. A continuacion se presentan los ítems analizados

- Padecimiento de ENT. Los profesionales de Contaduría Pública de la Universidad Santo Tomas tienen problemas de salud en lo que se conoce como enfermedades no transmisibles, tal como se muestra en el gráfico 1, ya que el $77.6 \%$ presenta alguna de estas enfermedades de modo que la aplicación de una guía de hábitos y estilos de vida saludable serviría de mucha ayuda a disminuir el padecimiento de estas enfermedades.

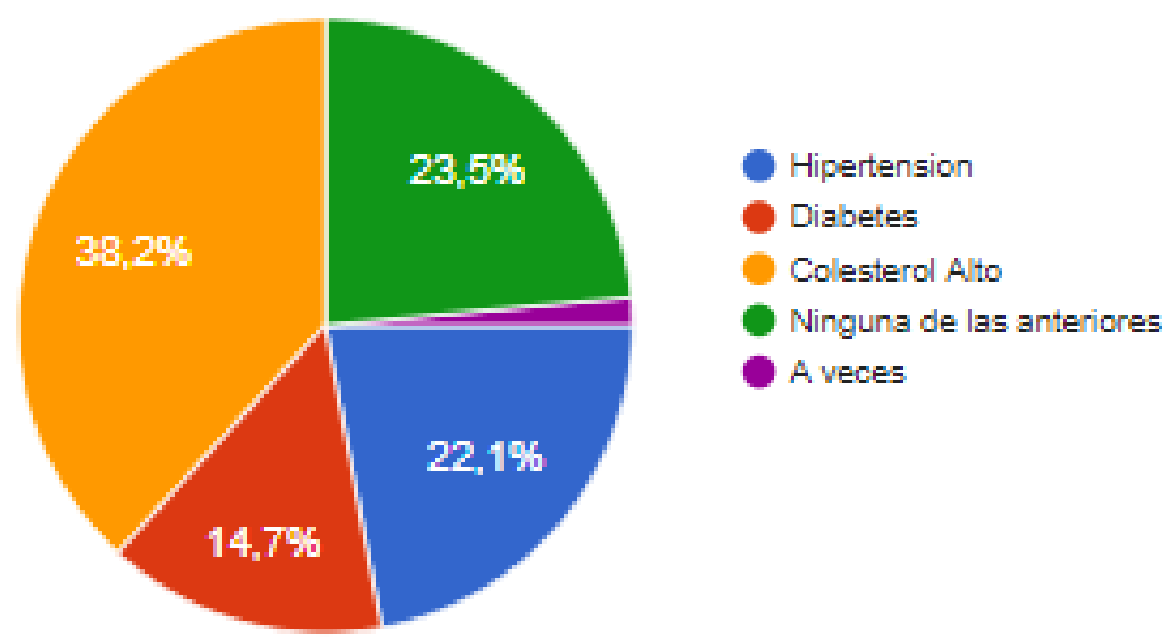

Gráfico 1. ¿Padeces alguna de estas enfermedades? por Daniel Andrés Garzón Céspedes, 2019

- Antecedentes Familiares. Hay un riesgo enorme en los egresados de la Universidad Santo Tomas en tener problemas asociados al corazón, pues un poco más de la mitad de los encuestados siendo exactos el 55.4\% en su núcleo familiar tanto de 1 grado 2 o 3 grado padecieron de ataques cardiacos. Una alimentación balanceada con unas pautas de ejercicios seria de mucha ayuda a que los egresados tengan una probabilidad menor de tener un ataque cardiaco ya que los hábitos asociados a buena alimentación y actividad física disminuyen la posibilidad de tener un ataque al corazón, como se puede evidenciar en el gráfico 2. 


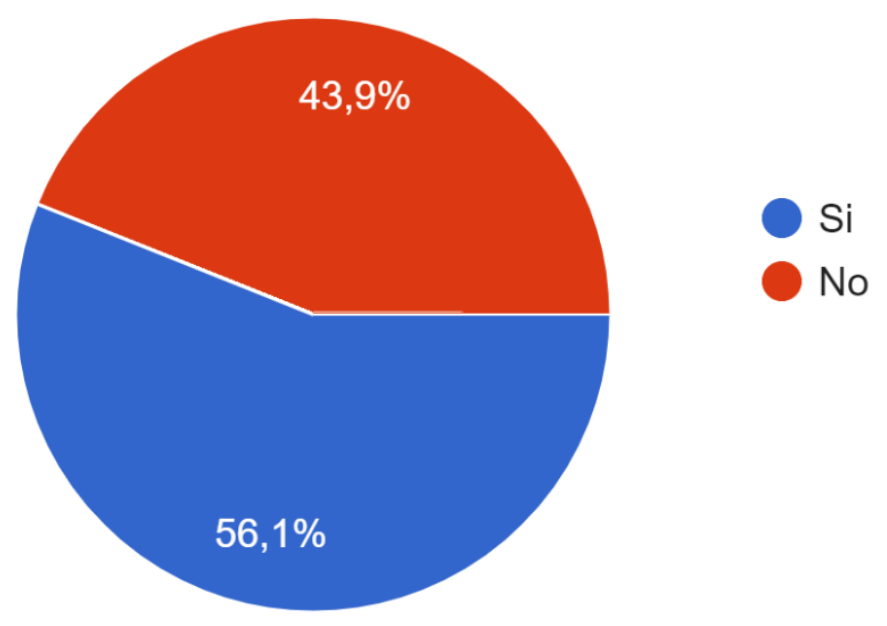

Gráfico 2. ¿Tienes antecedentes familiares de infarto? por Daniel Andrés Garzón Céspedes, 2019

- Consumo de Alimentos Saludables. Los egresados del programa de Contaduría Pública consumen muy poca fruta, como se muestra en el gráfico 3, pues el $13.6 \%$ de los encuestados no consume fruta y el $43.9 \%$ consume entre 0 y 3 porciones siendo un consumo aún muy bajo, ya que se estipula en la OMS un consumo de frutas de más de 4 porciones. Un consumo óptimo de frutas ayuda a disminuir el desarrollo o padecimiento de enfermedades no transmisibles como colesterol alto y triglicéridos que afectan ya dicho a varios de los egresados.

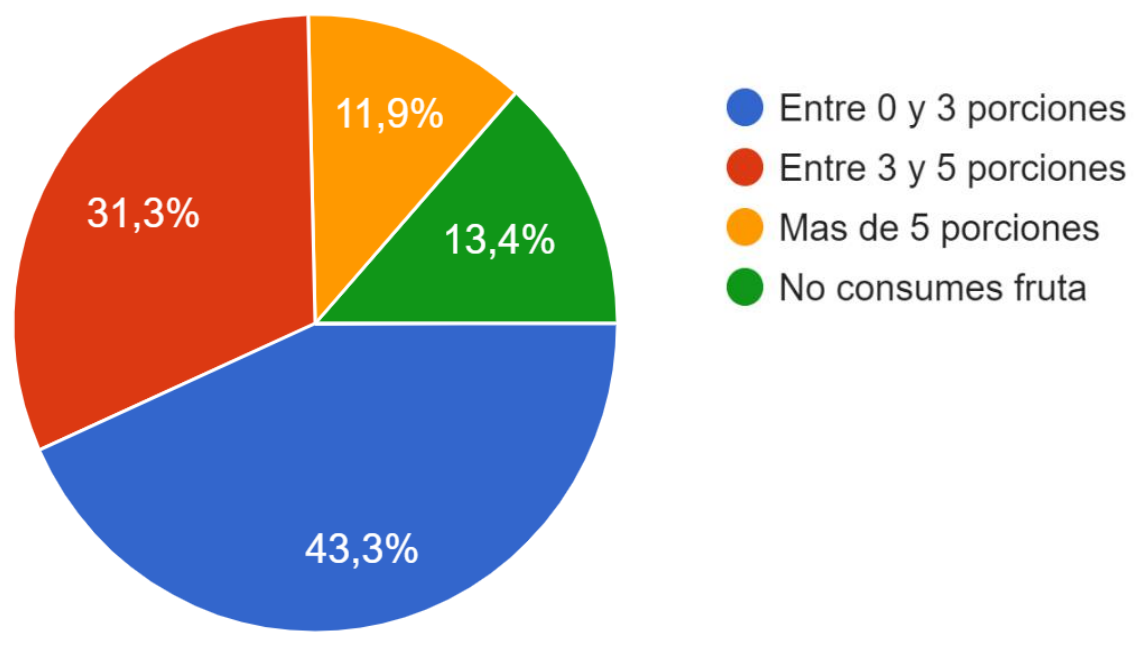

Gráfico 3. ¿Cuánta fruta consumes al día? por Daniel Andrés Garzón Céspedes, 2019 
- Pausas Activas. El panorama no es alentador en el ítem de actividad física en la vida laboral de los egresados de Contaduría Pública pues desafortunadamente en varios de los lugares de trabajo de nuestros encuestados no se realizan pausas activas, pues en solo un $12.3 \%$ si realizan pausas activas, el $18.5 \%$ en algunas ocasiones y más de la mitad de los encuestados en sus lugares de trabajo no conocen o no se aplican un programa mínimo de pausas activas, todo lo anterior se puede evidenciar en el gráfico 4.

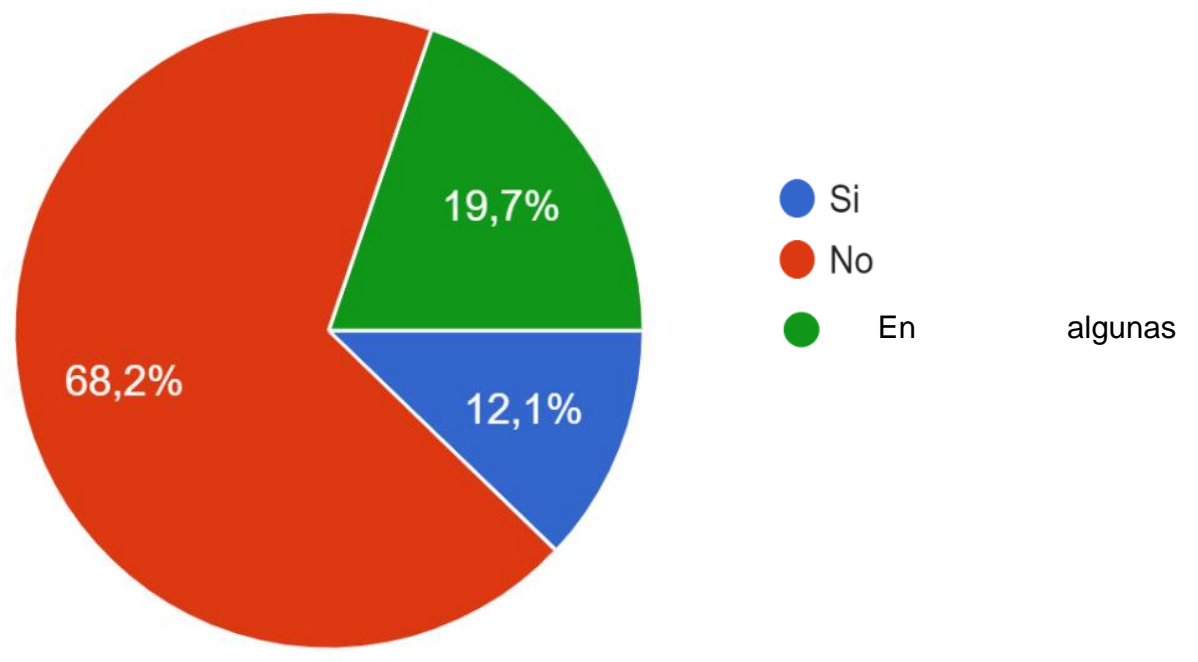

Gráfico 4. ¿En tu jornada laboral realizas pausas activas? por Daniel Andrés Garzón Céspedes, 2019

- Consumo de Nutrientes. En cuanto al consumo de verduras los profesionales de Contaduría Pública de la Universidad Santo Tomas de Villavicencio, las cifras de las encuestas, evidenciadas en el gráfico 5, dicen que el consumo de verduras es muy bajo pues tan solo el $4.5 \%$ de los encuestados consume verduras todos los días y entre 3 y 4 veces a la semana tan solo el $23.9 \%$ 


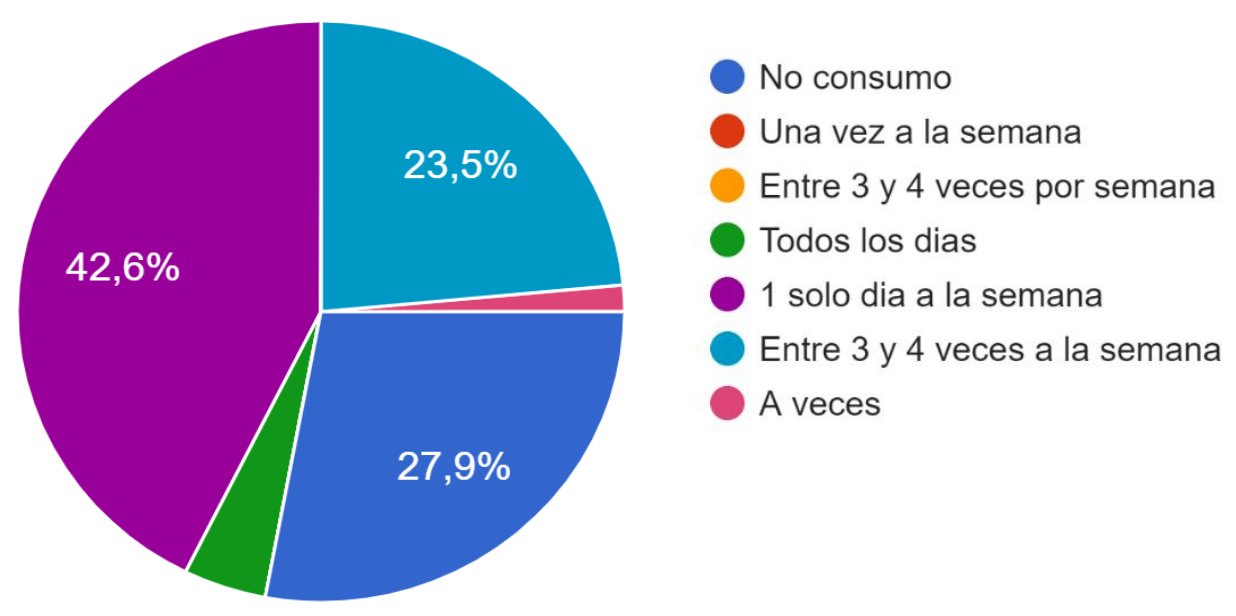

Gráfico 5. ¿Cuántas veces consumes verduras a la semana? por Daniel Andrés Garzón Céspedes, 2019

- Hidratación. Un consumo de agua mínimo, que se muestra en el gráfico 6, hacen los profesionales de Contaduría Pública de la Universidad Santo Tomas, pues tan solo tienen un consumo óptimo de agua el 38\% del total de los encuestadas, donde en un panorama negativo hay profesionales que consumen muy poca agua como lo reflejan 7 personas del total de los encuestados, cabe resaltar que un consumo óptimo de agua ayuda al cuerpo a mantenerlo hidratado y también ayuda a disminuir el desarrollo de enfermedades no transmisibles y a no tener problemas de salud.

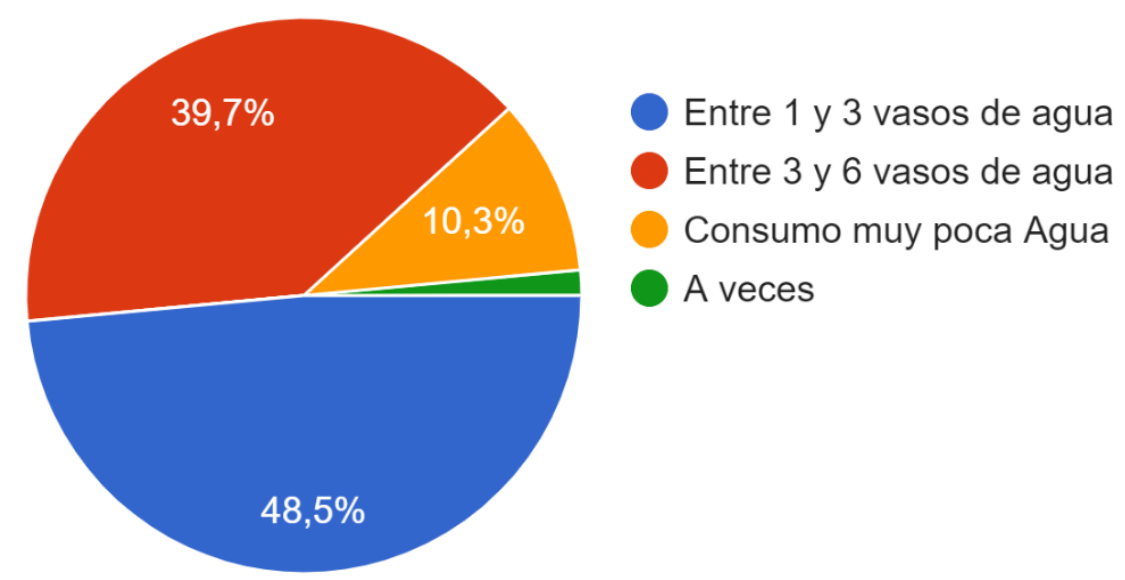

Gráfico 6. ¿Qué cantidad de agua consumes al día? por Daniel Andrés Garzón Céspedes, 2019 
- Tiempo en Familia. En la vida profesional como en lo personal es importante compartir con los seres queridos o en su defecto con alguna persona de tu vida social, en el caso de los egresados del programa de Contaduría Pública de la Universidad Santo Tomas no es así del todo, tal como se aprecia en el gráfico 7, ya que casi el $23 \%$ de los encuestados no comparte con sus seres queridos al menos 2 horas al día y el $38.8 \%$ a veces lo hace, como se puede evidenciar en el siguiente gráfico.

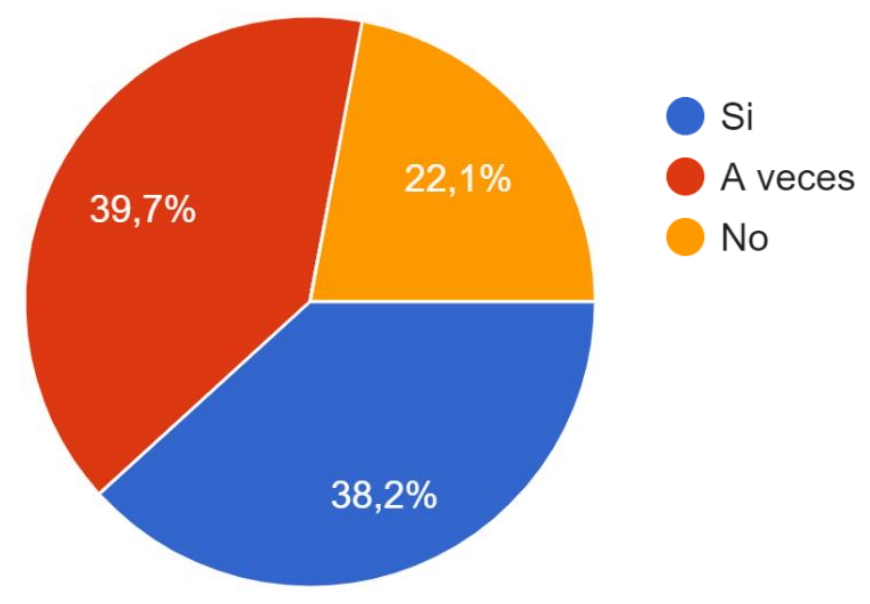

Gráfico 7. ¿Compartes con tus seres queridos al menos dos horas diarias? por Daniel Andrés Garzón Céspedes, 2019

- Horas de Sueño. La importancia del sueño es un factor fundamental para el desempeño laboral y la calidad de vida de las personas, infortunadamente los profesionales de Contaduría Pública duermen muy poco, no se sabe a profundidad cual es el factor por el cual no duermen bien, lo cual se expresa en el gráfico 8, pero la verdad es que tan solo el $30.3 \%$ de los encuestados tienen buenas horas de sueño para recuperar energía y encaminar una vida más saludable y con riesgos menores de tener problemas de salud. 


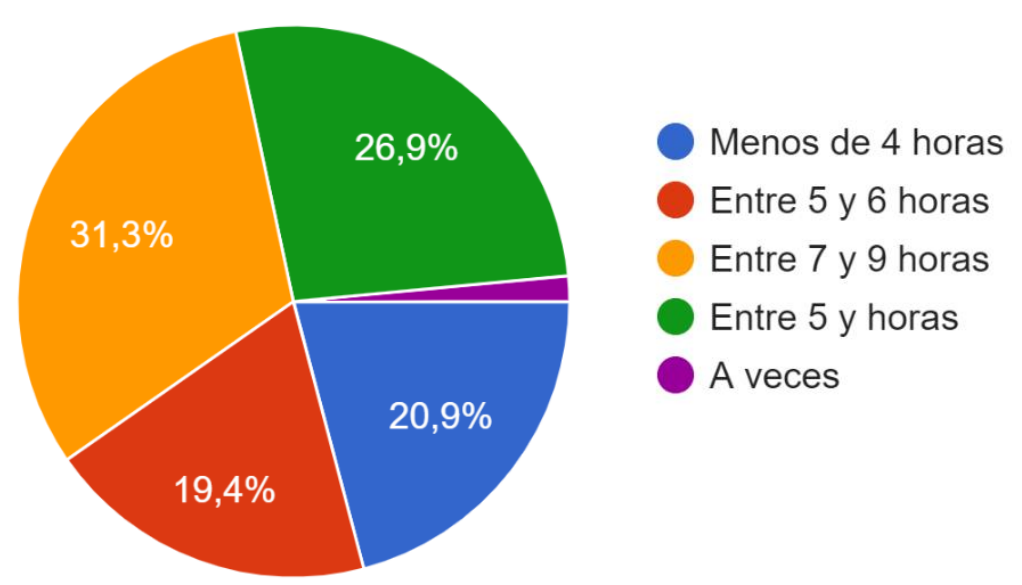

Gráfico 8. ¿Cuántas horas consideras que duermes al día? por Daniel Andrés Garzón Céspedes, 2019

- Hábitos Higiénicos. Los egresados de contaduría pública de la Universidad Santo Tomas de Villavicencio al momento de consumir algún alimento no se lavan las manos de manera frecuente, como se observa en el gráfico 9, pues tan solo 16 personas del total de los encuestados si lavan las manos al momento de consumir algún alimento; esto como factor de salud e higiene es importante ya que nos previene de infecciones y posibles enfermedades que deterioren la calidad de vida.

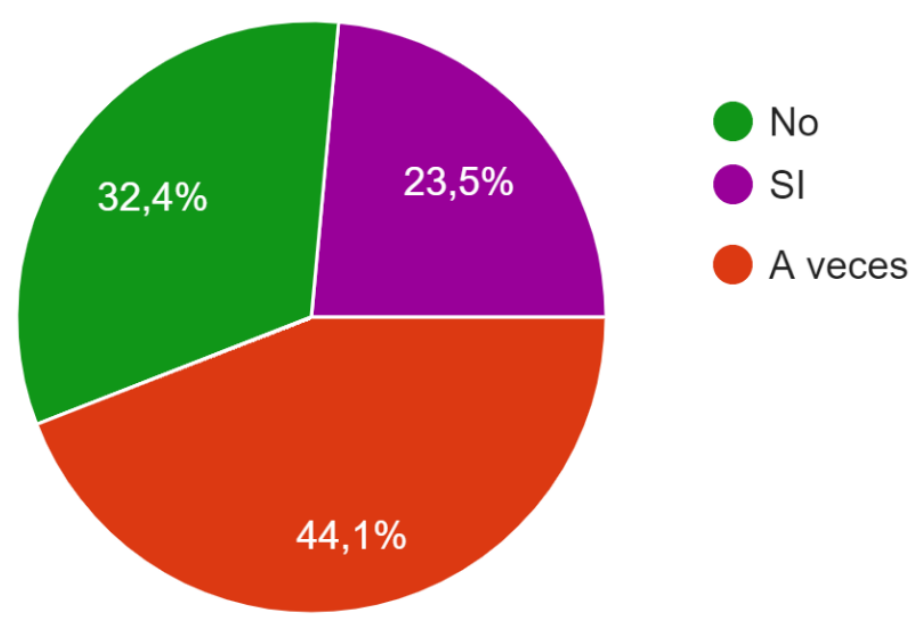

Gráfico 9. ¿Lava sus manos antes de consumir algún alimento? por Daniel Andrés Garzón Céspedes, 2019 


\section{Capítulo V. Conclusiones}

Una vez realizada la encuesta a los egresados se observó detalladamente que los hábitos y estilos de vida saludable de los profesionales de contaduría pública de la Universidad Santo Tomas de Villavicencio no son buenos, pues dentro de los nueve preguntas realizadas a los 68 profesionales en varias de ellas se evidencia por la tabulación de la encuesta que llevan una mala alimentación donde responden a un consumo mínimo de 3 porciones de frutas que es lo mínimo que se recomienda comer para llevar una buena calidad de vida, con en un porcentaje del $57.5 \%$.

En otra consulta que se realizó con el tema de alimentación, los Contadores Públicos tomasinos comen muy pocas verduras, tan solo el $4.5 \%$ consume verduras todos los días. También se comprobó que los profesionales de Contaduría Pública consumen muy poca agua que es vital para una salud optima desafortunadamente las cifras nos dicen que el $62 \%$ de los encuestados tienen un consumo menor al recomendado para funciones vitales y de calidad de vida optimas.

La encuesta afirma que comparten muy poco con sus seres queridos dentro de su jornada laboral o amigos con un porcentaje del $61.2 \%$. Siendo esta una de las preguntas con mayor impacto negativo. En otra pregunta donde se califica que tantas horas de sueño tienen los profesionales se evidencia en las encuestas que el 69. 7 \% duerme menos de lo recomendado y desde allí se puede tener trastornos emocionales y una calidad de vida negativa afectando tanto en la laboral como en lo personal.

El 69.2\% de los egresados de Contaduría Pública dentro de sus empresas no realizan pausas activas ocasionando un sedentarismo que al pasar los días será perjudicial para la salud. Sumado a estos factores negativos de alimentación, horas de sueño, consumo de agua y poco compartir con sus seres queridos, los profesionales de Contaduría Pública tienen antecedentes altos de sufrir un ataque cardiaco y padecer de enfermedades crónicas no transmisibles siendo esta una pregunta más desde un factor secundario si no se toman medidas de precaución como mejorar la calidad de vida; puede llevar a problemas más serios en un futuro afectando mortalmente la vida de los Contadores Públicos. 


\section{Capítulo VI. Producción Esperada}

Mediante las recopilaciones de datos encuestas e implementación de las mismas se busca que mediante una guía práctica y fácil de entender el Contador Público se puede adentrar a un mundo de vida más saludable y por ende que sus niveles de vida mejoren, mediante ejercicios básicos que ayuden a que toda la sociedad Contable sea una sociedad más competitiva desde los estándares de salud y con esto que un Contador Público sea lo suficientemente eficaz en su compañía y genere un plus adicional de las otras ramas administrativas y sea lo suficientemente vigoroso para la implementación de otras ramas y así se pueda dar un valor en términos de salud que ayude a un país que tiene grandes problemáticas en cuanto a niveles de salud concierne desde sus profesiones o estilos de vida de oficina.

Es de vital importancia hacer tomar conciencia a la comunidad de los profesionales de las Ciencias Contables que un buen nivel de calidad de vida es importante para el desarrollo y perfeccionamiento como ser, ya que nos permite estar más activos y saludables creando así una relación salud - trabajo en donde no solo se mejora la salud de nosotros sino a su vez un mejor desempeño en el campo laboral. 


\section{Capítulo VII. Propuesta de Intervención. \\ Estrategia Pedagógica}

A continuación se presenta una estrategia pedagógica planteada a partir de los resultados obtenidos en la presente investigación y denominada "Hábitos y Estilos de Vida Saludables" (Apéndice C), con la cual se busca brindar a la comunidad de los Contadores Públicos una herramienta que les permita facilitar la obtención y ejecución de manera cotidiana de pautas prácticas y de sencilla ejecución, que propendan el logro de estilos de vida saludables, transformando así su presente y futura vida, de modo tal, que la interacción con su entorno familiar, laboral y social sea armonioso y saludable.

La estrategia pedagógica denominada "Hábitos y Estilos de Vida Saludables" contiene parámetros asociados a la salud, la higiene, la actividad física, la alimentación balanceada, y la importancia de la vida en comunidad. Dentro de los ítems que maneja, se presentan

- Pautas para tener una alimentación balanceada y saludable.

- Una guía nutricional.

- Ejemplos para realizar pausas activas.

- Ejercicios de movilidad y articulación de movimiento que se pueden desarrollar durante la jornada laboral.

- Rutinas de ejercitación.

- Relevancia de la hidratación.

- Importancia en la asignación de tiempo y espacio para la vida familiar, la participación en grupos sociales y en equipos de trabajo. 


\section{Capítulo VIII. Recomendaciones}

No es una petición, es una necesidad que los profesionales de Contaduría Pública Tomasinos mejoren sus condiciones laborales en el entorno de alimentación, pausas activas, vida social porque si no se lleva una calidad de estilos de vida saludables a tiempos menores o en un futuro se puede presentar problemas serios de salud más de los que se están ya evidenciando.

Se recomienda llevara a cabo un programa de alimentación, ejercicio o pausas activas que mejoren las condiciones de vida de los contadores públicos, bajo la implementación del manual de hábitos y estilos de vida saludable ya que la salud es el principal factor de vida del ser humano y sin ella no se puede alcanzar nada. 


\section{Referencias}

Acuña, Y., \& Cortes, R. (Abril de 2012). Promociòn de Estilos de vida saludable en el àrea de salud de Esparza. Obtenido de Instituto Centroamericano de Administracion Publica: http://biblioteca.icap.ac.cr/BLIVI/TESIS/2012/acuna_castro_yessika_sa_2012.pdf

Albertman, E. (2 de Marzo de 2017). Día Mundial del Agua: La importancia del consumo y preservación. (L. Tercera, Entrevistador) Obtenido de www2.latercera.com: http://www2.latercera.com/noticia/dia-mundial-del-agua-la-importancia-consumopreservacion/

Álvarez, F. (2010). Sedentarismo y Actividad Física. Finlay - Revista Médica Clentífica de Cienfuegos, 55-60.

Álvarez, G., \& Andaluz, J. (2015). Estilos de vida saludable en los estudiantes internos de enfermería de la Universidad de Guayaquil 2014 - 2015. Obtenido de Universidad de Guayaquil:

http://repositorio.ug.edu.ec/bitstream/redug/8193/1/TESIS\%20ESTILOS\%20DE\%20VID A\%20SALUDABLE\%20EN\%20INTERNOS\%20DE\%20ENFERMERIA\%20UG.pdf

Bennasar-Veny, M. (2012). Estilos de Vida y Salud en Estudiantes Universitarios: La universidad como entorno promotor de la salud. Obtenido de Universitat de les Illes Balears - Tesis Doctorales en Xarxa: https://www.tesisenred.net/handle/10803/84136

Buckley, J., Hedge, A., Yates, T., Copeland, R., Loosemore, M., Hamer, M., . . Dunstan, D. (2015). The sedentary office: an expert statement on the growing case for change towards better health and productivity. British Journal Of Sports Medicne, 1357-1362.

Cabezas-Zabala, C., Hernández-Torres, B., \& Vargas-Zárate, M. (2016). Aceites y Grasas: Efectos en la salud y regulación mundial. Revista de la Facultad de Medicina de la Universidad Nacional, 761-768.

Cáez-Ramírez, G., \& Casas-Forero, N. (2007). Formar en un estilo de vida saludable: otro reto para la ingenierìa y la insdustria. Pedagogìa Universitaria - Universidad de La Sabana, 103-117. 
Caracol Radio. (2016). Màs de un millòn de colombianos pueden tener diabetes y no saberlo. $\begin{array}{llll}\text { Obtenido } & \text { de } & \text { Caracol }\end{array}$ http://caracol.com.co/radio/2016/04/08/salud/1460071947_112292.html

Carrillo-Mora, P., Ramírez-Peris, J., \& Magaña-Vázquez, K. (2103). Neurobiología del sueño y su importancia: Antropología para el estudiante universitario. Revista de la Facultad de Medicina de la UNAM, 5-15.

Casado-Pèrez, C., Hernàndez-Barrera, V., Carrasco-Garrido, P., \& Palacios-Ceña, D. (2015). Physical activity in adult working population: Results from the European National Health Survey for Spain . Atenciòn Primaria - Sociedad Española de Medicina de Familia y Comunitaria , 563-572.

Choque, R. (2005). Comunicaciòn y Educaciòn para la promociòn de la salud. Obtenido de Razòn y Palabra: http://www.razonypalabra.org.mx/libros/libros/comyedusalud.pdf

Cigarroa, I., Sarqui, C., \& Zapata-Lamana, R. (2016). Efectos del sedentarismo y obesidad: Una revisión de la actualidad Latinoamericana. Universidad y Salud, 156-169. Obtenido de http://www.scielo.org.co/pdf/reus/v18n1/v18n1a15.pdf

COLDEPORTES. (2008). decreto 2771 de 2008. Obtenido de Departamento Administrativo del Deporte, la Recreación,la Actividad Físcia y el Aprovechamiento del Tiempo Libre COLDEPORTES:

http://www.coldeportes.gov.co/sala_prensa/noticias_coldeportes/actividad_fisica_es_poli tica_estado

Cortés, M., Chiralt, A., \& Puente, L. (2005). Alimentos Funcionales: Una historia con mucho presente y futuro. Vitae - Revsita de la Facultad de Química Farmacéutica de la Universidad de Antioquia , 5-14.

Cuéllar-Montoya, Z. (2005). Pronunciamiento de laAcademia Nacional de Medicina frene al Proyecto 052/04 de Reforma a la Ley 100 de 1993. Revista de Medicina, 157-178.

Daza, M., \& Ramos, E. (2014). Estilos de vida saludable de los estudiantes de secundaria del Instituto Colombo - Venezolano de la ciudad de Medellín. Obtenido de Corporacion Universitaria Adventista: htthttp://repository.unac.edu.co/handle/11254/68

DeÀguila, R. (2012). $4^{a}$ Joranda ¿Còmo enfrentar las Enfermedades No Transmisibles desde la Multisectorialidad? Obtenido de Organizaciòn Panamericana de la Salud - Ministerio de Salud de Chile: http://www.paho.org/chi/images/PDFs/4foro\%20_2_.pdf?ua=1 
Dietz, W. (1998). Health consequences of obesity in youth: childhood predictors of adult disease. Pediatrics, 518-525.

Escobar, I., \& Rossi, A. (2007). Sobrepeso y obesidad incrementan la epidemia de diabetes tipo 2. Obtenido de EnColombia: https://encolombia.com/medicina/revistas-medicas/ddiabetes/d22/sobrepeso-y-obesidad/

Explorable. (2018). El Muestreo en la Investigación . Obtenido de https://explorable.com/es/muestreo-discrecional?gid=1694

Fernández, C., \& Suárez, R. (2017). El peso de ser un país que se engordó. El Tiempo. Obtenido de https://www.eltiempo.com/vida/salud/colombia-esta-en-camino-a-tener-una-epidemiade-obesidad- 155328

FunEsCo. (2018). Hipertensión. Obtenido de Fundaciòn Española del Corazón: https://fundaciondelcorazon.com/prevencion/riesgo-cardiovascular/hipertension-tensionalta.html

García, A., Martínez, M., \& García, A. (2008). Enfermedades Metabólicas de aparición en la edad adulta. Analos Sistema Sanitario de Navarra, 75-89. Obtenido de http://scielo.isciii.es/scielo.php?script=sci_arttext\&pid=S1137-66272008000400006

Grimaldo, M. (3 de Septiembre de 2010). Calidad y estilo de vida saludable en un grupo de estudiantes de posgrado de la ciudad de Lima. Pensamiento Lógico, 17-38.

Hernández-Sampieri, R., Fernández, C., \& Baptista, M. (2014). Metodología de la investigación. México: McGraw Hill.

Herrero, J. (2011). Estilo de vida y clima laboral. Obtenido de Equipos \& Talento: https://www.equiposytalento.com/tribunas/hay-group/estilo-de-vida-y-clima-laboral

HHS. (2018). Síntomas de un ataque cardíaco. Obtenido de United States Department of Health and Human Services - Office On Women's Health: https://espanol.womenshealth.gov/heart-disease-and-stroke/heart-disease/heart-attackand-women/heart-attack-symptoms

Jalisco. (2012). Estrategia Estatal para la Prevenciòn y el control del Sobrepeso, la Obesidad y la Diabetes. Obtenido de Gobierno del Estado de Jalisco: https://www.gob.mx/cms/uploads/attachment/file/22244/EstrategiaSODJalisco.pdf 
Loaiza, B., \& Peña, A. (2013). Niveles de estrés y sindrome de Burnout en contadores públicos colombianos. Actualidad contable FACES., 27-44. Obtenido de http://www.redalyc.org/articulo.oa?id=25728399003

Manes, F. (13 de octubre de 2015). ¿Para qué nos sirve dormir? (E. País, Entrevistador) Obtenido de https://elpais.com/elpais/2015/10/12/ciencia/1444635682_009779.html

MEN. (2009). Ley 1355 de Octubre 14 de 2009. Obtenido de Ministerio de Educación Nacional de Colombia: https://www.mineducacion.gov.co/1759/w3-article-381525.html

Mendieta, F., \& Salgado, C. (2013). Deporte en el contexto laboralde la empresa en producción. Obtenido de Universidad del Valle: http://bibliotecadigital.univalle.edu.co/bitstream/10893/4275/1/DEPORTE\%20EN\%20E L\%20CONTEXTO\%20LABORAL\%20DE\%20LA\%20EMPRESA\%20DE\%20PRODU $\mathrm{CCI} \% \mathrm{C} 3 \% 93 \mathrm{~N} . \mathrm{pdf}$

MinSalud. (2007). Ley 1122 de 2007. Obtenido de Ministerio de Salud y Protección Social: https://www.minsalud.gov.co/sites/rid/Lists/BibliotecaDigital/RIDE/DE/DIJ/ley-1122de-2007.pdf

MinSalud. (2015). Pausas Activas. Obtenido de Ministerio de Salud y Protecciòn Social: https://www.minsalud.gov.co/sites/rid/Lists/BibliotecaDigital/RIDE/VS/PP/ENT/abecepausas-activas.pdf

MinSalud. (2017). Dìa Mundial de la Hipertensiòn Arterial. Obtenido de Ministerio de Salud y Protecciòn Social de Colombia: https://www.minsalud.gov.co/sites/rid/Lists/BibliotecaDigital/RIDE/VS/PP/ENT/diamundial-hipertension-2017.pdf

MinSalud. (2017). Encuesta Nacional de la Situaciòn Nutricional ENSIN. Obtenido de Ministerio de Salud y Protecciòn Social de Colombia: https://www.minsalud.gov.co/sites/rid/Lists/BibliotecaDigital/RIDE/VS/ED/GCFI/ensincolombia-2018.pdf

Monje, C. (2011). Metodología de la Investigación Cualitativa y Cuantitativa. Guía Didáctica. Neiva: Universidad Surcolombiana.

OMS. (2014). Informe sobre la Situaciòn Mundial sobre las Enfermedades No Transmisibles. Obtenido de Organización Mundial de la Salud: http://www.who.int/es/news-room/factsheets/detail/noncommunicable-diseases 
OMS. (2017). Diabetes: Datos, cifras y consecuencias. Obtenido de Organizaciòn Mundial de la Salud: http://www.who.int/es/news-room/fact-sheets/detail/diabetes

OMS. (2018). Alimentación Sana: Datos, cifras y Panorama General. Obtenido de Organizacion Mundial de la Salud: http://www.who.int/mediacentre/factsheets/fs394/es/

OMS. (2018). Inactividad física: Un problema de salud pública mundial. Obtenido de Organización Mundial de la Salud: https://www.who.int/dietphysicalactivity/factsheet_inactivity/es/

OMS. (2018). Nutrición y Alimentación Sana. Obtenido de Orgnización Mundial de la Salud: http://www.who.int/topics/nutrition/es/

ONU. (2000). Declaración del Milenio. Obtenido de Organizaciòn de Naciones Unidas: https://www.un.org/spanish/milenio/ares552.pdf

OPS. (2016). Guì de Entornos y Estilos de Vida Saludables en Comunidades Indigenas Lencas. Obtenido de Organizaciòn Panamericana de la Salud - OPS (PAHO): http://iris.paho.org/xmlui/bitstream/handle/123456789/34580/vidasaludable2016spa.pdf? sequence $=1 \&$ is Allowed $=\mathrm{y}$

Pedersen, D. (1998). El desarrollo humano y la salud mental: Perspectivas socioculturales y apuntes metodológicos. Obtenido de Biblioteca Virtual de Saùde - Sâo Paulo: http://pesquisa.bvsalud.org/sms/resource/pt/lil-666520

Pedersen, D., Huayllasco, E., \& Erràzuriz, C. (2007). Salud Mental de la Comunidad. Ayacucho: Universidad McGill - Universidad Cayetano Heredia.

Pèrez, M. (2014). Concepto y Clasificaciòn de la Obesidad. Obtenido de Dietistas \& Nutricionistas: $\quad$ https://www.dietistasnutricionistas.es/concepto-clasificacion-de-laobesidad/

Quintero, J. (2014). Hábitos alimentarios, estilos de vida saludables y actividad física en neojaverianos del programa Nutrición y Dietética de la PUJ - Primer Período 2014. Obtenido de Pontificia Universidad Javeriana: https://repository.javeriana.edu.co:8443/bitstream/handle/10554/16030/QuinteroGodoyJi nnethViviana2014.pdf? sequence $=1 \&$ isAllowed $=\mathrm{y}$

Reyes, S. (Julio de 2008). Factores que intervienen a llevar estilos de vidasaludables en el personal de enfermería en los servicios de medicina y cirugía en el Hospital San 
Benito,Petén. Obtenido de Universidad de San Carlos de Guatemala: http://biblioteca.usac.edu.gt/tesis/05/05_8580.pdf

Rosas, H., \& Siegel, P. (Marzo de 2011). Promoción de salud y estilos de vida saludables en jóvenes universitarios: Hacia un curriculum integral. Obtenido de Universidad Autral de Chile: http://cybertesis.uach.cl/tesis/uach/2011/ffr789p/doc/ffr789p.pdf

Sans-Menéndez, S. (2007). Enfermedades Cardiovasculares. Obtenido de Institut d' Estudis de la Salut Barcelona: http://www.mscbs.gob.es/organizacion/sns/planCalidadSNS/pdf/equidad/07modulo_06.p df

Schukitt, M. (1995). Drug and alcohol abuse: A clinical guide to diagnosis and tratment. New York: Plenum Press. Obtenido de http://www.ametep.com.mx/aportaciones_noviembre_estilosvida05.htm

Senado. (1991). Constitución Política de Colombia. Obtenido de htpp/www.senado.gov.co: htpp/www.senado.gov.co/sisjur/Norma1.jsp?i=4125

Talero-Gutiérrez, C., Durán-Torres, F., \& Pérez-Olmos, I. (2013). Sueño: Características Generales,patrones fisiológicos y fisiopatológicos en la adolescencia. Revista Ciencias en Salud, 333-348.

UDistrital. (2012). Estrategias Didácticas para el Aprendizaje Colaborativo. Obtenido de http://acreditacion.udistrital.edu.co/flexibilidad/estrategias_didacticas_aprendizaje_colab orativo.pdf

Vázquez, D. (2016). El Sedentarismo es un problema de salud pública. Obtenido de El Tiempo: http://www.eltiempo.com/archivo/documento/CMS-16556059

Velasco, M., \& Mosquera, F. (2007). Estrategias didácticas para el aprendizaje colaborativo. Obtenido de Universidad Distrital Francisco José de Caldas: http://acreditacion.udistrital.edu.co/flexibilidad/estrategias_didacticas_aprendizaje_colab orativo.pdf

Velázquez, S. (2012). Actividades Educativas para una vida saludable. Sakud fisica y Emocional. Pachuca: Universidad Autònoma del Estado de Hidalgo. 


\section{Apéndices}

\section{Apéndice A. Solicitud de Permiso}

Villavicencio, Marzo 26 de 2019

Doctor

\section{ORLANDO BERMUDEZ}

Médico Deportólogo

Asunto. Solicitud de permiso y viabilidad de aplicación de la encuesta

Reciba un cordial saludo respetado y apreciado doctor. La presente solicitud tiene como fin pedir cordialmente el visto de aceptación por parte de usted para la aplicación de una guía de hábitos y estilos de vida saludable que se piensa aplicar a los profesionales de contaduría pública de la universidad Santo Tomas de Villavicencio en un estudio de investigación realizado por mí, DANIEL ANDRES GARZON CESPEDES identificado con c.c. 1121881366 de Villavicencio estudiante de pregrado del programa de Contaduría Pública de la Universidad Santo Tomas de Villavicencio en mi tesis de pregrado acerca de cómo mejorar los habitos y estilos saludable del Contador Público desde su práctica profesional .

Solicito muy amablemente y con el conocimiento por parte de usted como profesional de medicina y especialista en Medicina del Deporte me de autorización para la aplicación de esta guía

Gracias por la atención prestada

Atentamente,

\section{Daniel Andrés Garzón Céspedes}

Estudiante de Contaduría Pública

Universidad Santo Tomas 


\section{Apéndice B. Encuesta ${ }^{1}$}

\section{Hábitos de vida del Contador Público Tomasino}

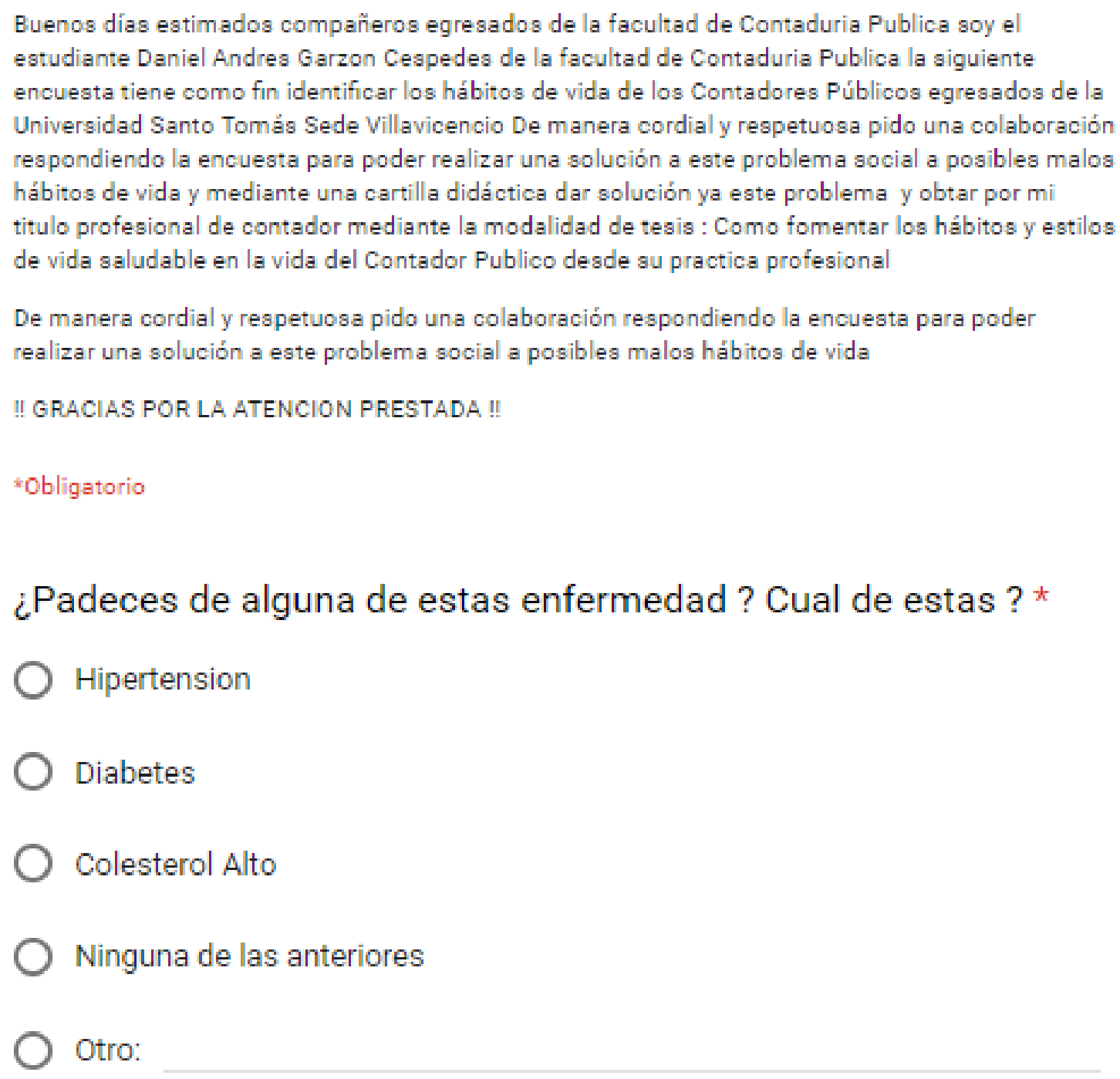

¿Tienes antecedentes familiares de infarto?

$\mathrm{OSi}$

$11 \quad$ Disponible en




\section{Apéndice C. Guía "Hábitos y Estilos de Vida Saludables. guía para Contadores Públicos}

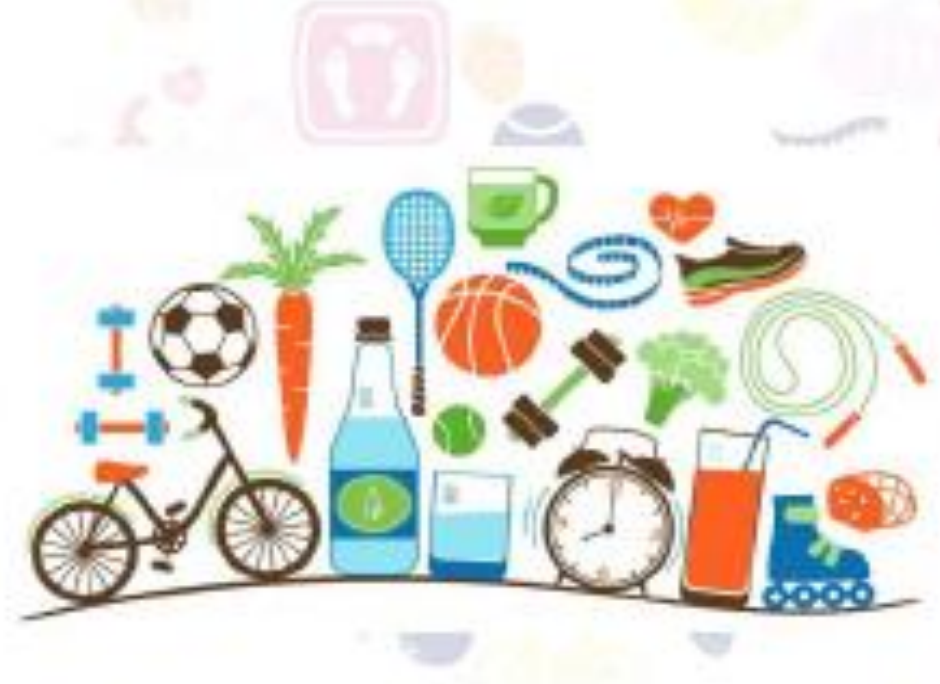

Hábitos y Estilos de Vida Saludables.

\section{Guía para Contadores Públicos}

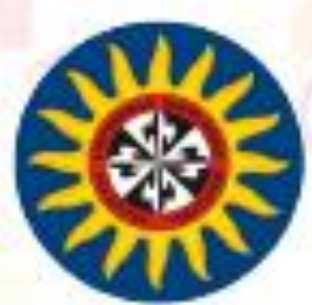

Daniel Garzón

Est. Contaduría Pública Universided Sento Tomas Villavicencio, 2019
Los hábitos y estiloe de vids saludable toman fuerza al pasar los sños, yo que es importante der prioridad a la saud; asi que medisnte is implementación de esta guia se pretende proporcionar pautas de sencilis resilización cotidiana que loe Contadores Públicos puedan mejorar su calided de vids, en entomos diversos, como el familiar, social y aboral; tomando como referente cuatro ejes temáticos:

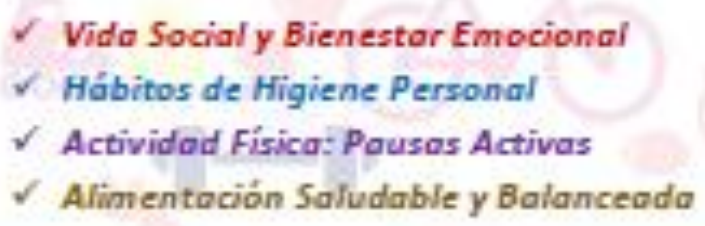

$\checkmark$ Vida Social y Bienestar Emocional

$\checkmark$ Hábitos de Higiene Personal

$\checkmark$ Actividad Fisica: Pausas Activas

$\checkmark$ Alimentación Saludable y Balanceada

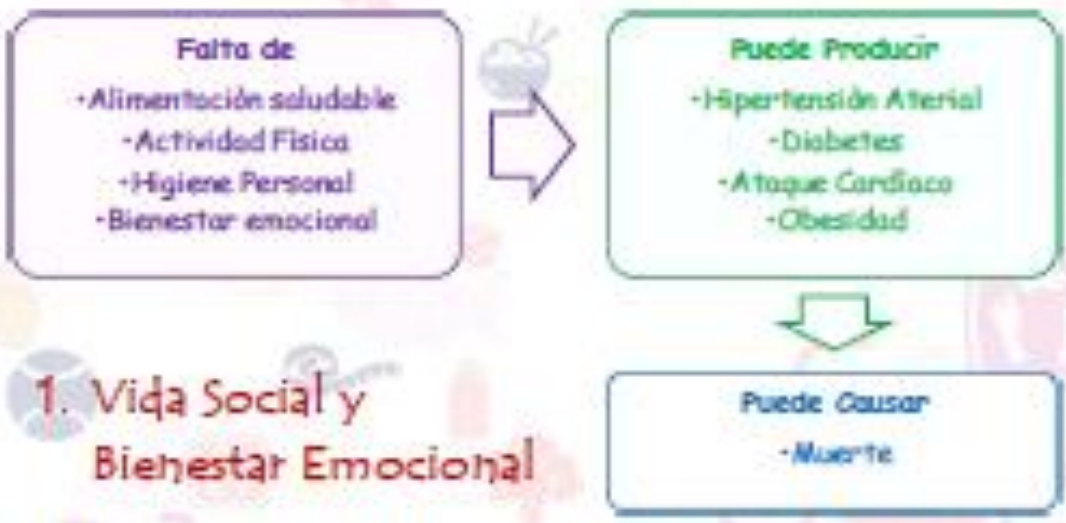

iEres de los que sólo se preocupan por el bienestar de los libros contables? ilos estados financieros? alos suditorias?

iPues haz una pausa en tu día!

Toma pauts que mejoren tu cotidianidsd y te den bienestar!

Teis gresenta que contar con una buena vida social trae mütiples beneffics fidoes emocionale para ti tu nicleb socid.

No todo en firabajel Ifrabejari IY Trabajel 


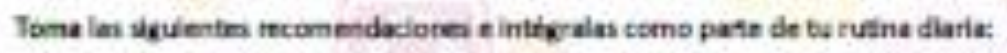

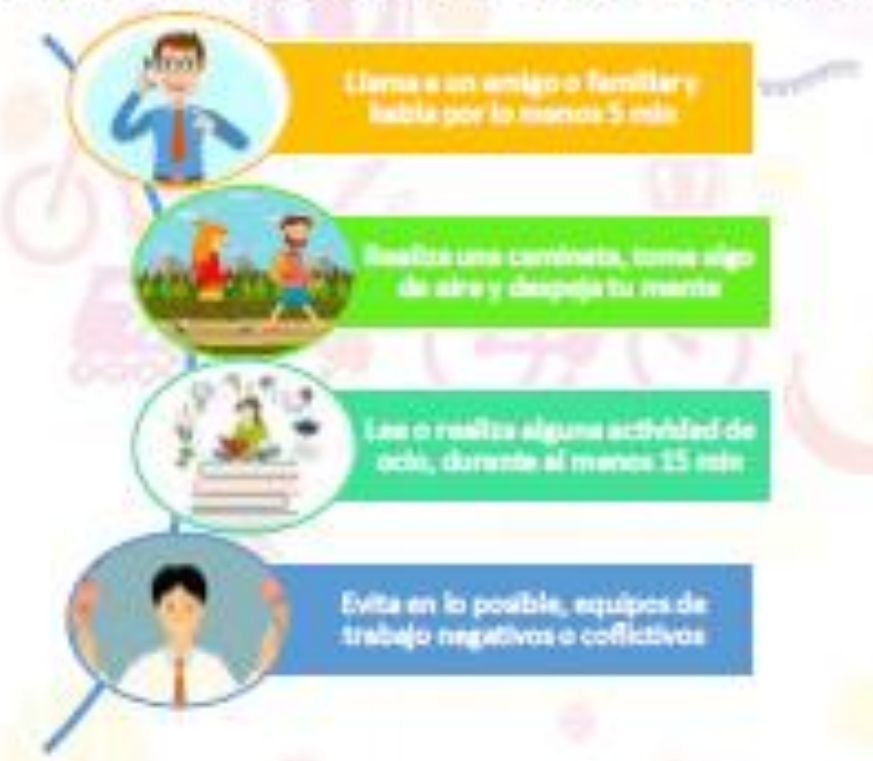

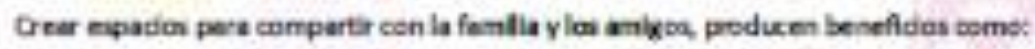

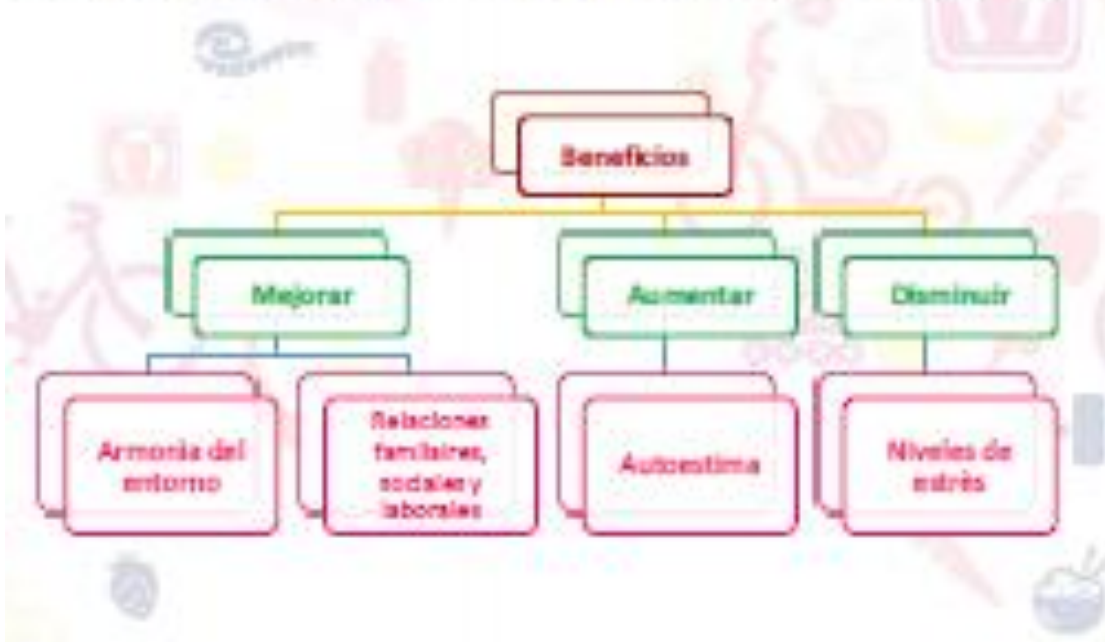

\section{Hábitos de Higiene Personal}

$$
\text { (1) }
$$

La sud e hodent come base fundamemal del equilbrlo del ser humane para of deienpefio de sus actividades darlas tiene, cuenta con itcricas part manterie una buene calliad de vida, ali que es importante terer en tuenta tres plares:

$$
\text { M P C }
$$

M. Majora lo buene que has hachu por bu salud, por ejemelo no soba lawarte "a

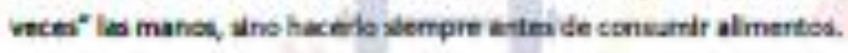

P. heverit shuaclones como la destidr atadin, mediante el comumo centante y

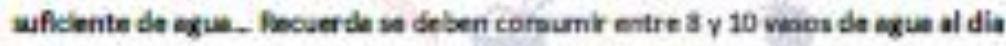

$$
\text { The }
$$$$
\text { varas }
$$

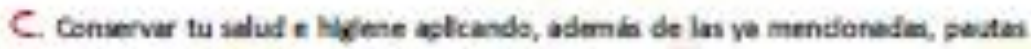
sereilles como las que aparnonh a coritnuadbh.

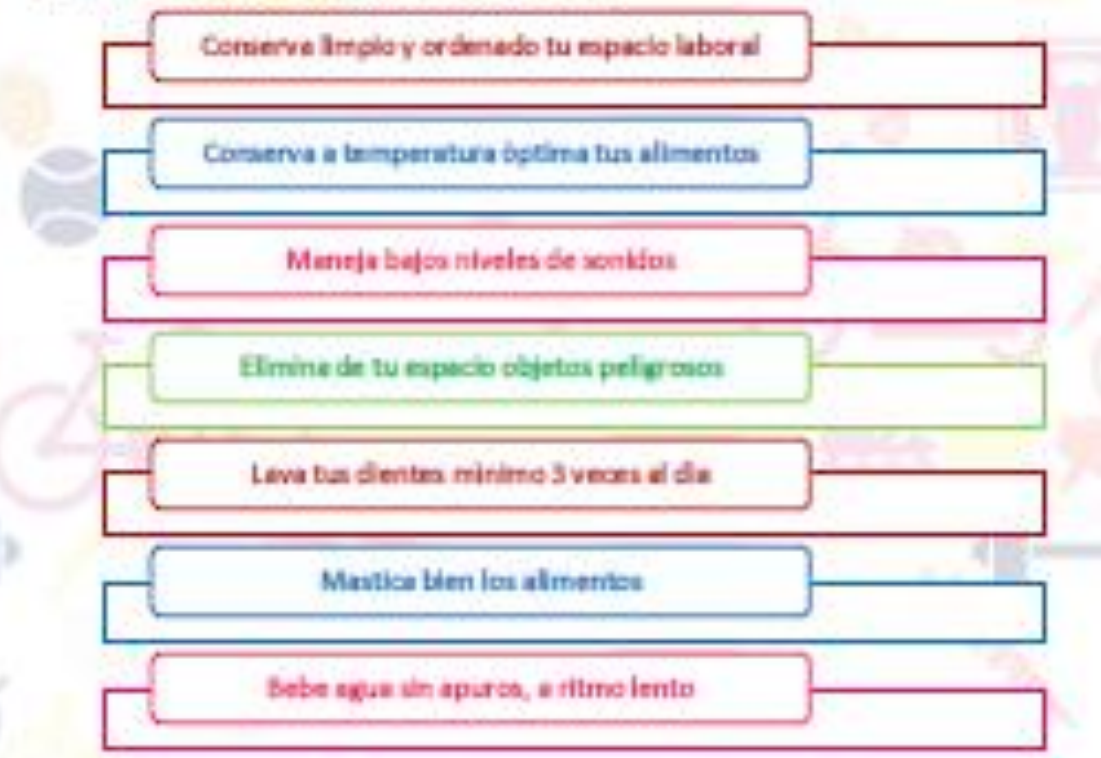




\section{Actividad Fisica: Pausas Activas}

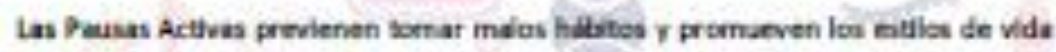

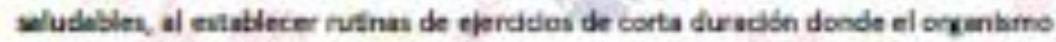

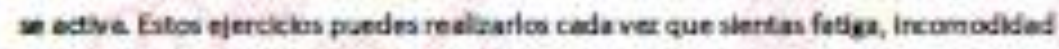
o dengaver, benefidindote de modo tal que w la haces, lo chtienes.
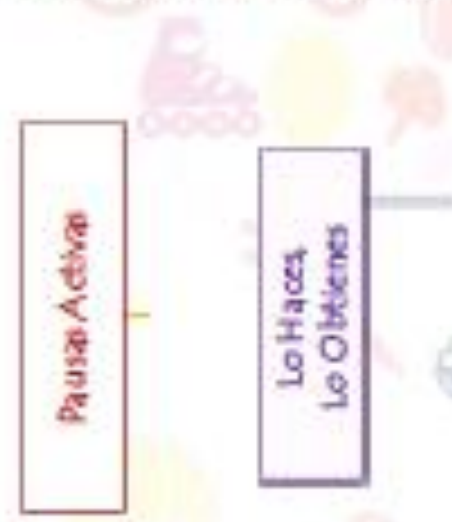

Ten preienta la importancla de realea mayor actividad fidca intex ede a ruthas semanales.

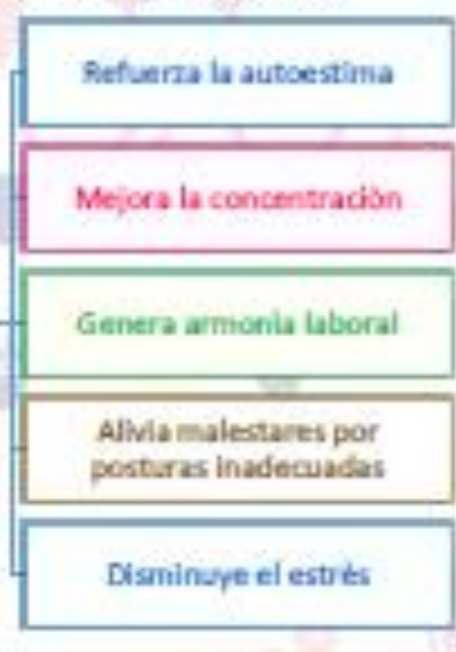

Se recomienda por lo menco que ien tres sesiones a la amana b:

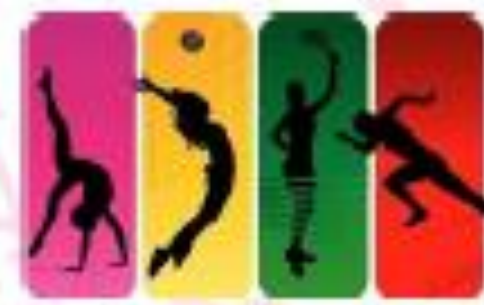

1 Ohnaviois

1 Contera de Acondidonamimto $\mathrm{Fblas}$

$\checkmark$ Deportes indidduales e en Corlumb

$\checkmark$ aale

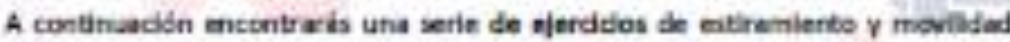

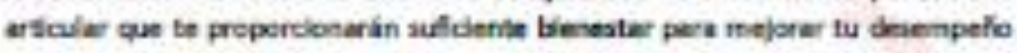
labord $\gamma$ warrentex tu celided de vidn, evitando situacienes nexativin como of sodentaxbme par causas laborale.
Movilidad Articular y Estiramiento
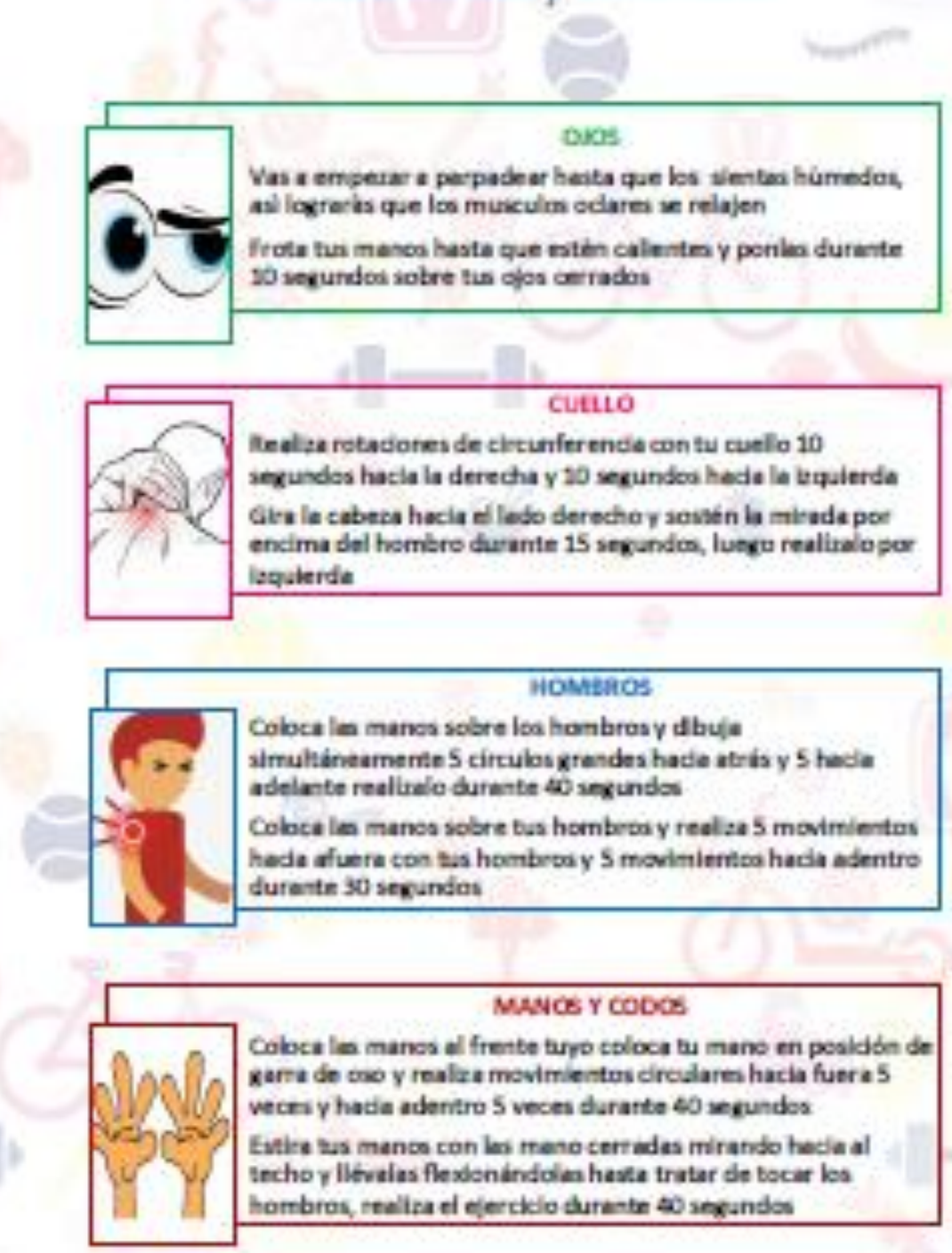

\section{Masics Y coDss}

Cobca le manos al frente turo coloca tu mano en posidibe de xarra de uno y realsa movimientersiculare hacia fueras vecris $y$ hadia adentru 5 veces durarta 60 seceundes

Gatire tus manos con las meno cerrides mirando heda a! Incho y literains flexdenindolan hasta tratar de bocar los hombras, realiza el ejercklo durantre so soxundas 

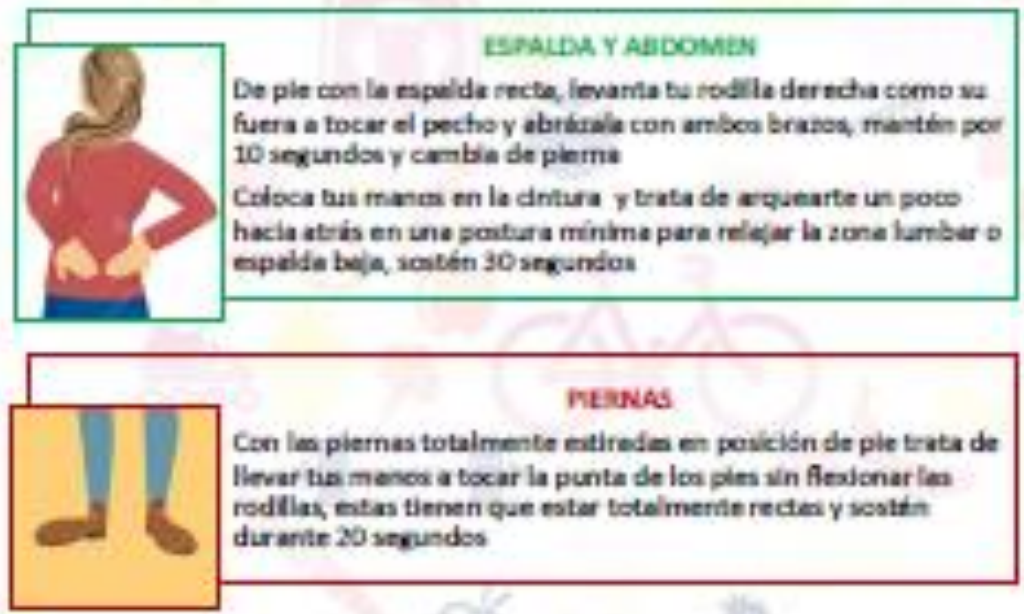

¿Y por quẻ no praebas estas rutinas?

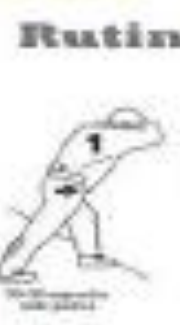

\section{1}
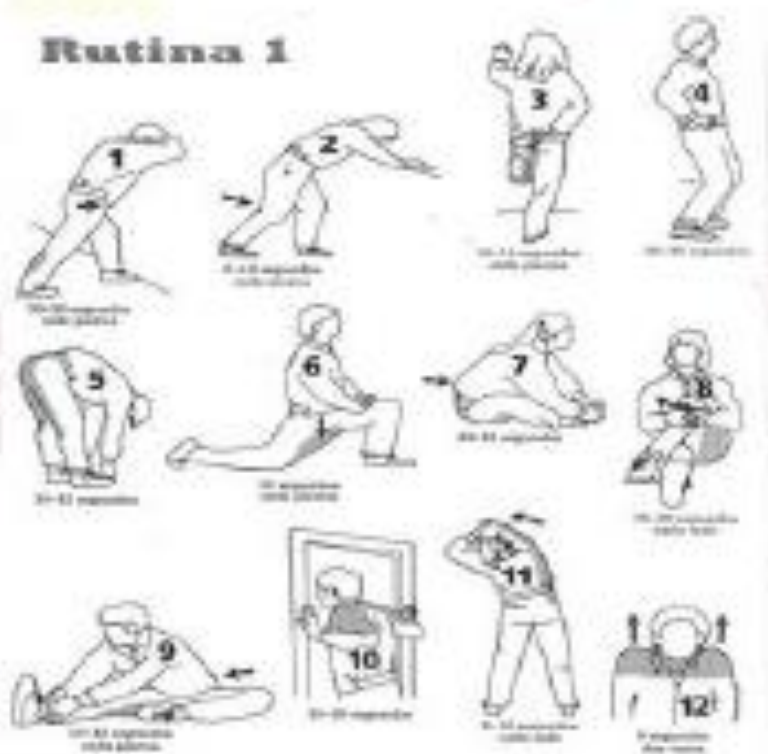

\section{Trateing 2}
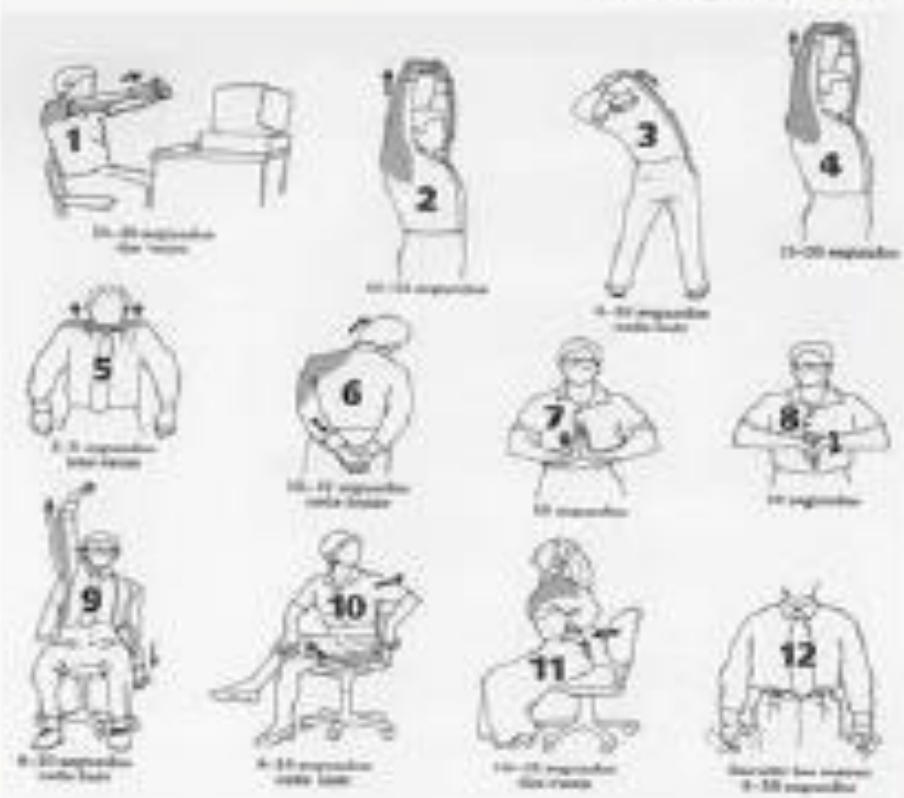

Ls anteriores rutinas de estiramiento son ficles y senelia de haces, solo debes detinar 10 minutes de te acenda.

\section{Alimentación Saludable y Balanceada}

Una dirtentadín saludabie, balanceada os la que indape mentalmente los

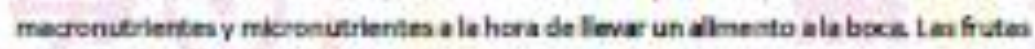

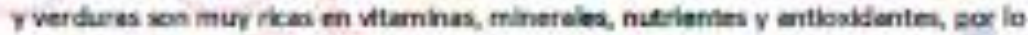
que son ldevins di quieres terer una elecueda nutidin.

El comumo dotimo de proteinas, xrinas y carbohidratso a requalto pera obterne une almentaclín balancreadec sobra dinct que si quieres mejorar tu caldad de ido es noctiario no convatrir extes almentens fritos. 


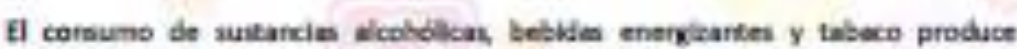
deterioru de la selud y obitaculae obtener bptimex teiultados al aplikar un plan de almantadón balanceade

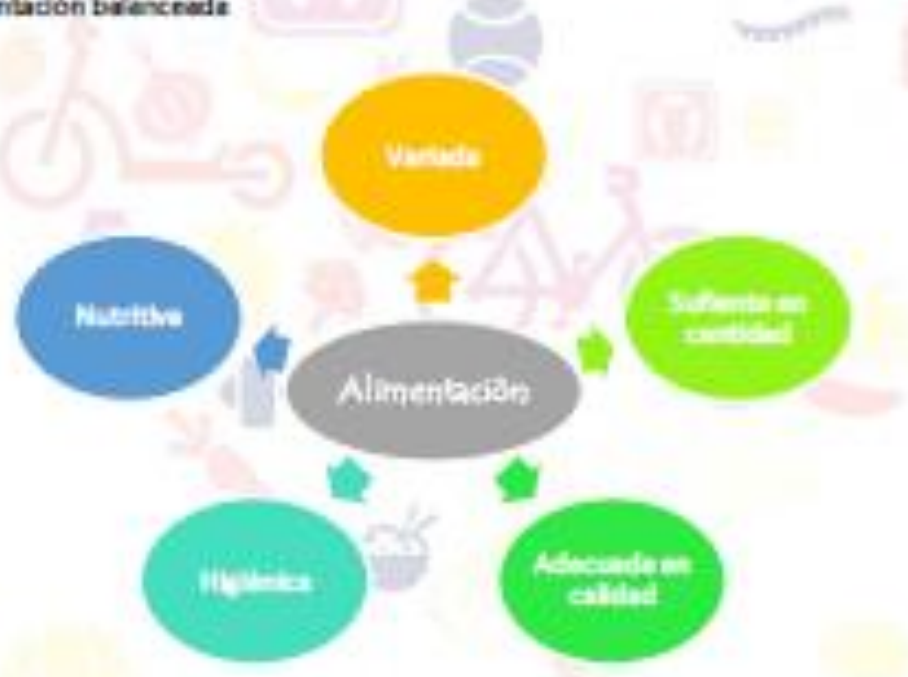

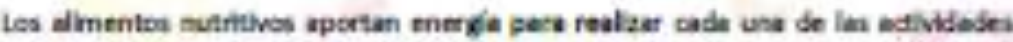
cotidinas y centienen de clases de nusiertex:

\section{Maoonutrientes. Como proteinan, carbolidratusy casas vexetile}

1. Micronutrientes. Como viarninas y minerales

A continaacidn entontraris une sia Nutriciond, cbo el fin de que pundan teme

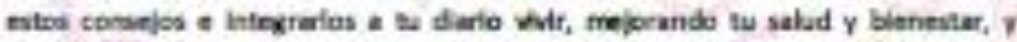

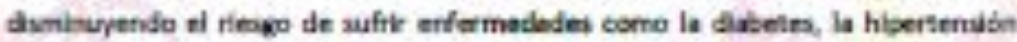
arterial, la Hperlpidenia y obesidad, entre otres, con almentes roxulados en calories, bajp conterido de sal y aucires rellhatos.

\section{DESAYUNO}

La idea en cbevamì alxin almento pere de cada itum de manera propordonal y en porciones,

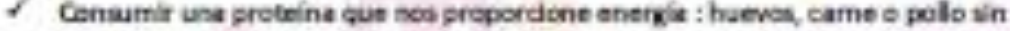
ser fritus o procmiados

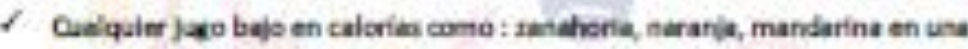
medida de 150 a 200 millties

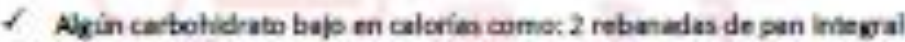

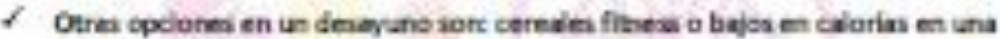
porelón de 150 xrimos con yoxurt bajs en atùca:

$$
\text { MEDIA MANANANA }
$$

(Consumir stlo una epdón al die)

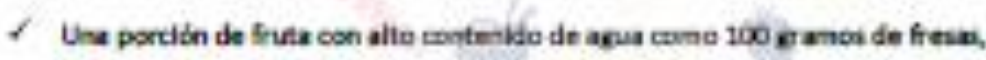
sandla, mel6n, KtwL.

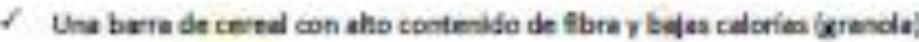

\section{Un yosurt griage}

7 Una pordibn de mani bajo en sodlo fau etqueta dobe mostra menos de $150 \mathrm{mel}$

\section{ALMUERZO}

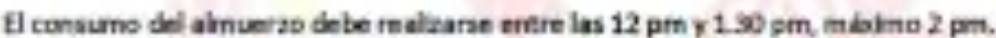

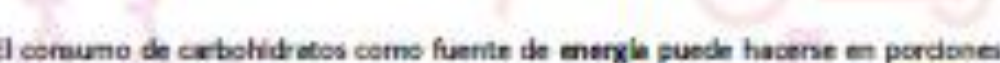
pequeño $y$ de un solo tiper, por sienpla, arree y no pape, papa y ho arror; freferblemente al vasor o codda, no frith.

A mornento decorsurrt protrinm animales o carnes, preferblemerte que seat ien

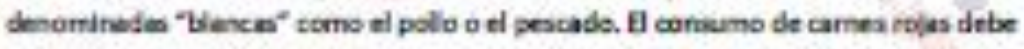
ier mbimo de dos portiones a la iemana en dos eportuntadedes diforentrs. Tambiben se recomienda que su porcitín ina acorde al peso corpord. For ajempla, sl pesas so

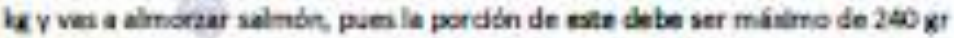

(30 belsena corpora * $3 \mathrm{~g}$ - Salmín = $240 \mathrm{~g}$ ) 
Ademb debes incluir ure porcién de vexptales, ye sean hortalin o verdurat. Se recorienda que ie consuman mbe 200 y $250 \mathrm{gr}$ de ensideda, que inclaye lechast,

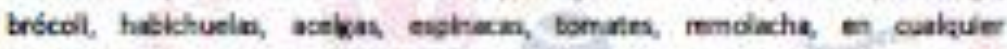
combinadicn, con adereiso naturale no wases corno el litnón.

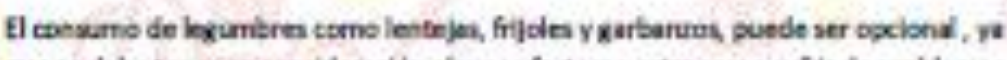

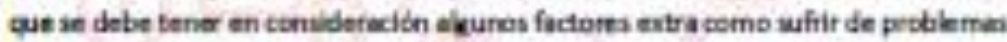
aleattros como calon ieritabie, entre otras.

\section{MEDLA TARDE \\ (Consumir sdlb una epción al dial)

$$
\text { 1) }
$$

El corauno de almentes en la jornide de la tarde, debe darse 3 horas despots del almuarzo, cen osdenes cume:

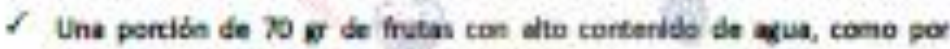
ejemplo antalie, Kuil, pilia, melón, saprya.

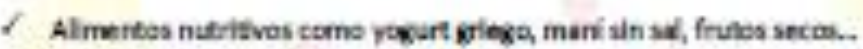

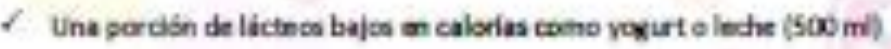

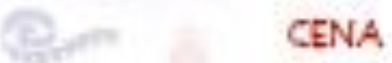

Ia thima comida del dia tene como bae furdartertal no inclur alinemteo cen alte

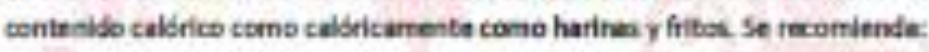

1 Cemurrir $100 \mathrm{~g}$ de vrketales y $100 \mathrm{~g}$ de probina arima al vaper

1. Un lictro bejo en colorias con un aperitivo coms mani

8 Un megaredafo de pan intesral baje en calorian won atuin y alyb de beber, como un juxo hatural sin erúcat

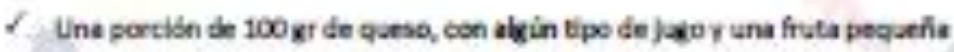

Tips ö́sicos pana una Alimentación Sana y Saludable

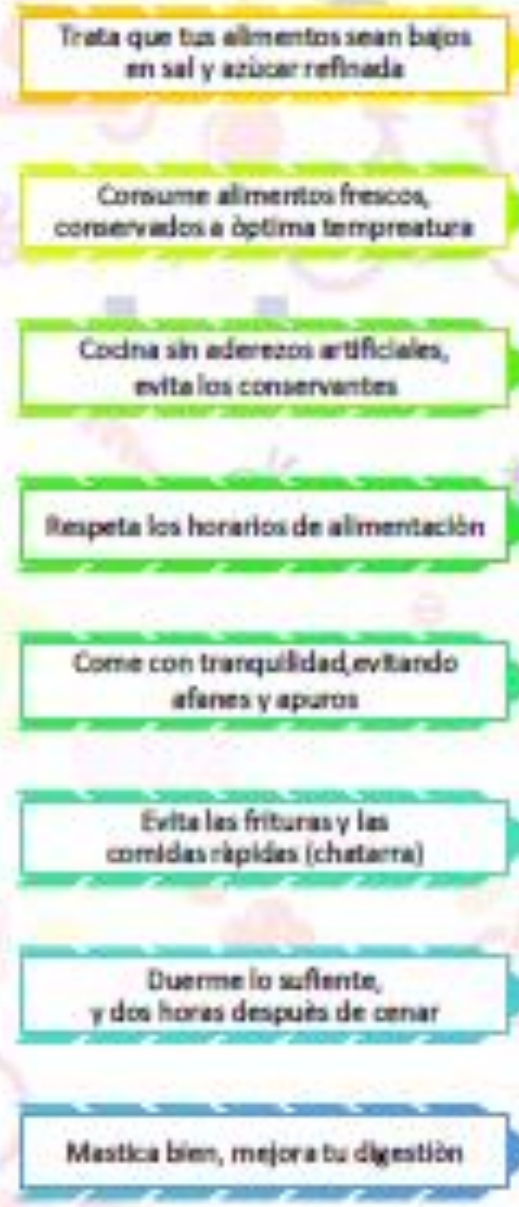

Una persona con häbitos y etílos de vida saladable, aporta significativamente al bienestar personal, comunitario y empresarial 